\title{
UNRAVELING THE ORIGINS OF NEARBY YOUNG STARS
}

\author{
VAleri V. MaKarov \\ Michelson Science Center, Caltech, Pasadena, CA; vvm@caltech.edu \\ Received 2006 June 6; accepted 2006 September 29
}

\begin{abstract}
A systematic search for close conjunctions and clusterings in the past of nearby stars younger than the Pleiades is undertaken, which may reveal the time, location, and mechanism of formation of these often isolated, disconnected from clusters and star-forming regions, objects. The sample under investigation includes $101 \mathrm{~T}$ Tauri, post-TT, and main-sequence stars and stellar systems with signs of youth, culled from the literature. Their Galactic orbits are traced back in time and near approaches are evaluated in time, distance, and relative velocity. Numerous clustering events are detected, providing clues to the origin of very young, isolated stars. Each star's orbit is also matched with those of nearby young open clusters, OB and TT associations and star-forming molecular clouds, including the Ophiuchus, Lupus, Corona Australis, and Chamaeleon regions. Ejection of young stars from open clusters is ruled out for nearly all investigated objects, but the nearest OB associations in Scorpius-Centaurus, and especially, the dense clouds in Ophiuchus and Corona Australis have likely played a major role in the generation of the local streams (TWA, Beta Pic, and Tucana-Horologium) that happen to be close to the Sun today. The core of the Tucana-Horologium association probably originated from the vicinity of the Upper Scorpius association $28 \mathrm{Myr}$ ago. A few proposed members of the AB Dor moving group were in conjunction with the coeval Cepheus OB6 association 38 Myr ago.
\end{abstract}

Subject headings: binaries: general — open clusters and associations: general — stars: kinematics

\section{INTRODUCTION}

Stars certainly do not form for no particular reason out of thin air. The nearest sites of ongoing star formation, e.g., in Orion and Corona Australis, display a wide array of dynamical processes in relatively dense gas and molecular cloud cores, and reprocessing of substantial amounts of energy released either via supernova explosions and high-velocity winds from massive stars or via cold gravitational collapse (Dolan \& Mathieu 2001). Observational evidence leaves little doubt that stars normally form in significant numbers nearly simultaneously, although generation of numerous low-mass stars may require the presence of a massive, shortlived star embedded in a dense cloud. The rough uniformity of the initial mass function over a set of open cluster and associations of different ages and the general field, supports the conjecture that new stars usually appear in large batches at once. It is all the more surprising that several dozens young stars (younger than the Pleiades, $\approx 100 \mathrm{Myr}$ ) identified within about $100 \mathrm{pc}$ of the Sun seem to either be isolated, detached from star formation regions, or come in sparse streams and loosely comoving groups, such as the TW Hya association (TWA in this paper), Beta Pictoris moving group (BETAPIC), Tucana-Horologium swarm (TUCHOR), $\mathrm{AB}$ Dor moving group (ABDOR), $\eta$ Cha cluster (ETACHA), $\epsilon$ Cha moving group, R CrA T-association, and HD 141569 group.

One may consider a heuristic explanation that an efficient dynamical process is responsible for ejection of stars from open clusters and associations. The merit of this conjecture is that no new theory is required to explain the existence of nearby young stars, since they could have formed in known clusters or OB associations and accidentally traveled to our neighborhood. Massive ejection of members is predicted from number simulations of dense, compact clusters during the short initial period of dynamical relaxation. One immediate difficulty arises from the high velocities of ejection (of order $10 \mathrm{~km} \mathrm{~s}^{-1}$ ) required to travel the distances separating the dispersed young stars and the nearest young clusters. Hard massive binaries can endow less massive single stars with considerable escape velocities on a close encounter, but few such binaries have been found in the near clusters of the age of Alpha Per (50 Myr) or younger. This model is not valid for sparse $\mathrm{OB}$ associations where the number density is too low for dynamical interactions with binaries to play a significant role. Sterzik \& Durisen (1995) proposed a mechanism of ejection of post-TT stars from nonhierarchical multiple systems (trapezia), which are inherently unstable. Simulations for this mechanism produce typical velocities in the range $1-3 \mathrm{~km} \mathrm{~s}^{-1}$, and ejected stars are expected to remain in the vicinity of their parent clusters for extended periods of time (Fukushige \& Heggie 2000). Alternatively, formation of minute stellar groups inside small, shortlived cloudlets dynamically dispersed from larger turbulent clouds was proposed by Feigelson (1996).

Before venturing into speculations on low-number star formation in sparse molecular clouds, we should carefully examine the simpler possibility of ejection. In this paper, I collect astrometry and kinematics data for 101 young stars, most of which are closer than $100 \mathrm{pc}$, proposed in the literature. Age determination for young stars is based on a number of observable parameters (none of which is self-sufficient), most notably, isochrone estimates for late-type stars, chromospheric activity, X-ray activity for solartype stars, equivalent width of lithium lines for late-type stars, and Herbig AeBe phenomenon for early-type stars. The sample is intended to include stars younger than the Pleiades $(\sim 100 \mathrm{Myr})$, but a few older stars can be included because of the ambiguity of age determination on isolated objects. The major compilation sources are Zuckerman \& Song (2004) and Wichmann et al. (2003); several chromospherically active stars with significant $\mathrm{Li}$ abundances are included from Strassmeier et al. (2000). Note that only stars with accurate parallaxes from the Hipparcos catalog and with reliable radial velocities are considered in this paper. Many more very young nearby stars have been identified in the literature but are left out of this analysis; for example, most of the proposed TWA members are missing in Hipparcos, and HAeBe stars in the $\epsilon$ Cha (Feigelson et al. 2003) and HD 141569 (Weinberger et al. 2000) groups are lacking accurate radial velocities. 
Using the epicycle approximation, described in detail in Makarov et al. (2004) three-dimensional (3D) orbits are calculated for each star on a 0.25 Myr grid between 0 (now) and -80 Myr in the past. The epicycle approximation is sufficiently accurate (within the uncertainty of the Oort constants $A$ and $B$ and the asymptotic vertical period $P_{\nu}$ ) over such limited time spans and for objects with low relative velocities (cf. the phenomenon of "Local Association" for young stars; Montes et al. 2001). These relatively simple models are used to compute the past heliocentric positions and velocities of the stars and clusters in question, properly taking into account the differential Galactic rotation and the quasiharmonic vertical oscillation. Asiain et al. (1999) utilize similar approximations to investigate the focusing phenomenon in the motion of the gravitationally unbound Pleiades moving group. For each star considered in this paper, close fly-bys with the other stars are sought for. Spatial proximity of young stars at a certain time in the past may reveal their common origin and the site of formation. Furthermore, similar kinematics data are culled for 43 clusters, associations, and star-forming clouds, their past orbits calculated and matched for intersections with those of the young stars. The latter technique is similar to the one used by Hoogerwerf et al. (2001) to trace back the origins of hot runaway stars, who used numerical integration for orbit reconstruction. It has been verified on a set of Ursa Major group members that the epicycle approximation and the integration algorithm by Hoogerwerf et al. (2001) produce consistent results. Based on the latter numerical integration code, a backtracking method has been applied by Ortega et al. (2002), de la Reza et al. (2006), and Jilinsky et al. (2005) to investigate the origins of the $\beta$ Pictoris, TWA, $\eta$ Cha, and $\epsilon$ Cha clusters.

Previously suggested association of young stars with various kinematic groups is given in Table 1 in the column labeled "Assoc. Previous." This information is mostly taken from Zuckerman \& Song (2004) and references therein, but the extended ABDOR membership from López-Santiago et al. (2006) and a few suggested members of the IC 2391 supercluster from Montes et al. (2001) are also taken into account.

\section{PHASE SPACE PARAMETERS}

Heliocentric coordinates $(X, Y, Z)$ and heliocentric velocities $(U, V, W)$, in the Galactic coordinate system, are given in Table 1 for 101 nearby young stars, along with radial velocities collected from the literature and parallaxes from the Hipparcos catalog. The $X$-axis is directed toward the Galactic center, the $Y$-axis toward the direction of Galactic rotation, and the $Z$-axis toward the north Galactic pole. The major source of uncertainty in the phase space parameters is imprecise radial velocities for some young stars, especially for spectroscopic and long-period astrometric binaries. Whenever possible, I use the center-of-mass radial velocities from complete spectroscopic orbital solutions (e.g., Torres et al. 2003). Parallaxes are always from the Hipparcos catalog; identified young stars missing in the Hipparcos catalog or those with failed astrometric solutions are not considered in this paper. Proper motions are usually taken from the Tycho-2 catalog (Høg et al. 2000), for a few stars, Hipparcos proper motions are utilized. The main sources of radial velocities are Nordström et al. (2004), Zuckerman \& Song (2004 and references therein), and Strassmeier et al. (2000).

Heliocentric coordinates, radial velocities, proper motions, distances, and phase space parameters for selected 43 open clusters, OB associations, T-associations, and star-forming regions are collected in Table 2. The main source of information for open clusters is Robichon et al. (1999), and for OB associations it is de Zeeuw et al. (1999). For several T-associations (e.g., Lupus,
R CrA, and $\rho$ Oph) I derived the $(U, V, W)$ velocities from the mean proper motions of carefully cross-identified members found in the UCAC2 catalog (Zacharias et al. 2004). Detailed analysis of the kinematic properties of these associations will be published elsewhere. Encounters between stars and clusters are displayed in Tables 3-15.

\section{THE TUCANA-HOROLOGIUM STREAM}

A group of comoving post-T Tauri stars in the Horologium constellation was discovered by Torres et al. (2000). They determined an approximate age of $30 \mathrm{Myr}$ for this sparse group and surmised that the actual size today could be larger than $50 \mathrm{pc}$. The mean velocity vector of the association as initially determined by Torres et al. (2000) is $(U, V, W)=(-9.5,-20.9,-2.1) \mathrm{km} \mathrm{s}^{-1}$. Within the same year, Zuckerman \& Webb (2000) proposed the Tucana association in the southern sky. It was subsequently realized that since the stars in Tucana and in Horologium have the same age and space motion, and occupy close positions in space, they are likely to be members of one extended and dispersed stream of young stars (Zuckerman et al. 2001a), abbreviated as TUCHOR in this paper. The number of proposed members of TUCHOR amounts to 49 now, but only those are included in this analysis that have reliable Hipparcos parallaxes and radial velocities ( Table 1). TUCHOR is well populated with G- and K-type stars and, therefore, shows up prominently in the Tycho-2/ROSAT sample of X-ray active stars (Makarov \& Urban 2001). Up to 49 stellar systems have been proposed for this group with a well-defined streamlike motion (Zuckerman \& Song 2004), but only 25 are included in this analysis (Table 1).

Figure 1 shows all pairwise approaches of proposed TUCHOR stars in the past 80 Myr. In this figure, as well as in Figures 2, 3, and 4 , the stars involved in encounters are specified in the rectangular blocks in the left-hand column. Each encounter event is depicted as a block with round corners, connected with horizontal and vertical lines to the pair of stars involved. Inside each event block, the minimum distance and the distance today are given in the upper line, in parsecs, the time of the closest approach in the lower left corner, in Myr, and the relative velocity at that time in the lower right corner, in $\mathrm{km} \mathrm{s}^{-1}$. Only 15 out of 25 proposed TUCHOR members appear to be closer to each other at some time in the past than they are now. The times of nearest approach, indicated with a minus sign in the lower left corner of each event block, range from -2.5 to -70 Myr. The simple arithmetic mean of the nearest approach times is -30.4 with a standard deviation of 22.4 Myr. The mean is quite close to the age (30 Myr) roughly estimated by isochrone fitting from theoretical evolution models for pre-main-sequence stars. However, the large spread of individual approaches in time and the modest number of stars involved suggest a weak degree of compression, if any, in the past. In other words, the group was not significantly smaller than it is today $(30-40 \mathrm{pc})$ at any time in the past. This conclusion is supported by low relative velocities at the nearest approach of, typically, 1 or $2 \mathrm{~km} \mathrm{~s}^{-1}$. TUCHOR is distinguished by its remarkably low degree of expansion, at least in the currently known boundaries.

Some stars have distinctly earlier encounters than the mean, for example, HIP 32435. This may be a clue that the group includes parts of somewhat different kinematic origin. Several core members were close to the Upper Scorpius progenitor cloud (US) 27 Myr ago ( Table 9), including the most reliable members HIP 107947 and 108195. The dispersion of closest approach times for this members is remarkably small. The relative fly-by velocities are also distributed compactly around $9.5 \mathrm{~km} \mathrm{~s}^{-1}$. Furthermore, at about the same time, and with slightly lower velocities, 
TABLE 1

Phase Space Parameters for Nearby Young Stars

\begin{tabular}{|c|c|c|c|c|c|c|c|c|c|c|c|}
\hline HIP & Name & $\begin{array}{c}\Pi \\
\text { (mas) }\end{array}$ & $\begin{array}{c}\mathrm{RV} \\
\left(\mathrm{km} \mathrm{s}^{-1}\right)\end{array}$ & $\begin{array}{c}X \\
(\mathrm{pc})\end{array}$ & $\begin{array}{c}Y \\
(\mathrm{pc})\end{array}$ & $\begin{array}{c}Z \\
(\mathrm{pc})\end{array}$ & $\begin{array}{c}U \\
\left(\mathrm{~km} \mathrm{~s}^{-1}\right)\end{array}$ & $\begin{array}{c}V \\
\left(\mathrm{~km} \mathrm{~s}^{-1}\right)\end{array}$ & $\begin{array}{c}W \\
\left(\mathrm{~km} \mathrm{~s}^{-1}\right)\end{array}$ & $\begin{array}{c}\text { Assoc. } \\
\text { (Previous) }\end{array}$ & $\begin{array}{c}\text { Assoc. } \\
\text { (This Paper) }\end{array}$ \\
\hline 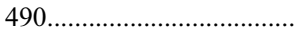 & & 24.9 & 1.6 & 10.6 & -5.5 & -38.4 & -10.0 & -21.5 & -1.3 & TUCHOR & TUCHOR(L) \\
\hline 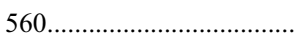 & HR 9 & 25.6 & 3.1 & 4.5 & 5.8 & -38.4 & -11.1 & -15.5 & -6.8 & BETAPIC & BETAPIC? \\
\hline 1113 & & 22.9 & 8.8 & 19.2 & -26.1 & -29.4 & -8.9 & -19.4 & -1.6 & TUCHOR & TUCHOR \\
\hline $1481 \ldots$ & & 24.4 & 6.6 & 15.5 & -19.0 & -32.8 & -8.9 & -19.9 & -0.9 & TUCHOR? & TUCHOR \\
\hline $2485 \ldots$ & & 19.0 & 9.8 & 18.6 & -24.8 & -42.7 & -12.3 & -25.5 & -2.7 & TUCHOR & $?$ \\
\hline 2578 & & 21.5 & 7.5 & 16.3 & -22.0 & -37.6 & -9.5 & -21.5 & -0.9 & TUCHOR & TUCHOR(L) \\
\hline 2729 & & 21.8 & -1.0 & 15.6 & -21.2 & -37.7 & -12.4 & -17.3 & 5.8 & TUCHOR? & $?$ \\
\hline $3586 \ldots \ldots \ldots \ldots \ldots \ldots .$. & & 20.6 & -14.8 & -25.6 & 40.8 & -6.7 & -9.1 & -25.8 & -15.3 & ABDOR & ABDOR? \\
\hline $6485 \ldots \ldots \ldots$ & HD 8558 & 20.3 & 8.3 & 10.5 & -23.0 & -42.3 & -10.2 & -21.9 & -0.3 & TUCHOR & TUCHOR(L) \\
\hline 6856 & HD 9054 & 26.9 & 7.1 & 5.8 & -15.5 & -33.3 & -8.7 & -19.2 & -0.5 & TUCHOR & $?$ \\
\hline 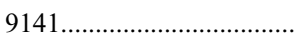 & HD 12039 & 23.6 & 5.7 & -11.1 & -3.5 & -40.7 & -10.6 & -21.0 & -1.2 & TUCHOR & TUCHOR \\
\hline 9892 & & 19.9 & 9.5 & 4.2 & -24.4 & -43.6 & -9.8 & -20.6 & -0.3 & TUCHOR & TUCHOR \\
\hline 9902 & HD 13246 & 22.2 & 10.5 & 7.3 & -24.8 & -36.8 & -10.2 & -20.3 & -1.2 & TUCHOR & TUCHOR \\
\hline $10272 \ldots \ldots \ldots \ldots \ldots \ldots$. & & 31.0 & -0.3 & -21.7 & 14.8 & -18.7 & -8.1 & -28.0 & -12.2 & ABDOR & ABDOR \\
\hline 10679 & & 29.4 & 4.8 & -24.0 & 16.9 & -17.2 & -10.7 & -13.6 & -8.0 & BETAPIC & BETAPIC \\
\hline $10680 \ldots \ldots$ & & 25.4 & 4.8 & -27.8 & 19.6 & -20.0 & -12.2 & -16.4 & -8.6 & BETAPIC & BETAPIC \\
\hline 11437 & & 23.7 & 6.7 & -31.1 & 20.9 & -19.5 & -14.4 & -15.9 & -8.6 & BETAPIC & BETAPIC \\
\hline 12394 & $\epsilon$ Hyi & 21.3 & 6.0 & 10.6 & -31.0 & -33.7 & -10.8 & -16.3 & 3.3 & TUCHOR & $?$ \\
\hline 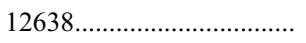 & & 19.9 & -4.2 & -39.1 & 26.8 & -16.6 & -8.2 & -28.1 & -13.3 & ABDOR & ABDOR \\
\hline 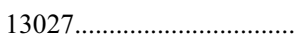 & & 30.7 & 3.7 & -24.4 & 10.3 & -19.0 & -7.6 & -27.4 & -11.4 & ABDOR & ABDOR \\
\hline $14551 \ldots \ldots \ldots \ldots \ldots \ldots$ & HD 19545 & 17.4 & 12.4 & -21.4 & -19.5 & -49.8 & -10.8 & -19.9 & -1.9 & $?$ & TUCHOR? \\
\hline 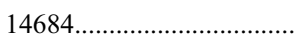 & HD 19668 & 24.9 & 14.6 & -23.7 & -4.7 & -32.1 & -5.1 & -28.8 & -10.3 & ABDOR & $?$ \\
\hline $14807 \ldots \ldots \ldots \ldots \ldots \ldots$. & & 19.4 & 4.1 & -42.2 & 14.8 & -25.9 & -5.4 & -29.3 & -16.2 & ABDOR & ABDOR? \\
\hline 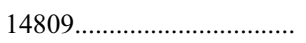 & & 20.2 & 4.1 & -40.4 & 14.2 & -24.7 & -5.3 & -28.0 & -15.6 & ABDOR & $?$ \\
\hline $15247 \ldots \ldots \ldots$ & HD 20385 & 20.0 & 7.2 & -33.2 & -3.0 & -37.2 & -8.8 & -20.7 & -0.1 & TUCHOR & ? \\
\hline 16563 & HD 21845 & 29.6 & -5.0 & -28.9 & 16.8 & -4.7 & -6.3 & -25.4 & -15.8 & $?$ & ? \\
\hline 16853 & HD 22705 & 24.0 & 14.4 & -4.4 & -25.8 & -32.5 & -9.3 & -20.3 & -1.1 & TUCHOR & TUCHOR \\
\hline $17928 \ldots \ldots \ldots \ldots \ldots \ldots \ldots \ldots$ & HD 23965 & 26.9 & 12.2 & -33.2 & 6.9 & -15.2 & -20.9 & -23.8 & 4.9 & TAUAU & $?$ \\
\hline 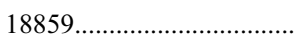 & GJ 159 & 52.0 & 18.1 & -15.1 & -2.9 & -11.5 & -8.3 & -28.8 & -12.2 & ABDOR & ABDOR? \\
\hline 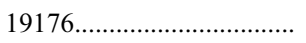 & & 6.4 & 15.5 & -142.0 & 17.4 & -60.9 & -14.3 & -9.8 & -9.1 & TAUAU & $?$ \\
\hline $19183 \ldots \ldots$ & & 18.1 & 15.9 & -44.7 & -7.5 & -31.6 & -5.4 & -27.3 & -13.6 & ABDOR & ? \\
\hline 19335 & GJ 9145 & 46.9 & 24.8 & -19.8 & 7.1 & -3.8 & -31.3 & -16.8 & -7.7 & $?$ & ? \\
\hline 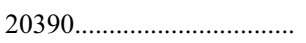 & $\mathrm{T}$ Tauri & 5.7 & 24.6 & -164.7 & 10.9 & -63.0 & -24.8 & -15.3 & -6.8 & TAUAU & TAUAU? \\
\hline 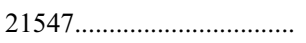 & 51 Eri & 33.6 & 21.0 & -24.3 & -8.2 & -15.2 & -14.0 & -16.2 & -10.1 & BETAPIC & BETAPIC \\
\hline $21852 \ldots \ldots \ldots \ldots \ldots \ldots \ldots \ldots$ & & 8.7 & 14.1 & -111.7 & 12.9 & -25.1 & -12.9 & -7.0 & -10.9 & TAUAU & ? \\
\hline 22295 & & 16.5 & 11.5 & 20.6 & -47.2 & -31.6 & -9.9 & -19.2 & 0.2 & TUCHOR & ? \\
\hline 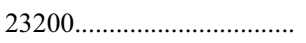 & GJ 182 & 37.5 & 32.4 & -23.3 & -7.4 & -10.7 & -23.6 & -20.9 & -14.9 & IC 2391 & $?$ \\
\hline 23309 & & 38.1 & 17.8 & -1.5 & -20.8 & -15.9 & -10.7 & -15.4 & -8.2 & BETAPIC? & ? \\
\hline $24244 \ldots \ldots \ldots \ldots \ldots \ldots$ & $\iota$ Lep & 13.5 & 25.0 & -55.3 & -35.5 & -33.9 & -13.8 & -23.9 & -7.0 & $?$ & TUCHOR(L)? \\
\hline 24947 & & 21.9 & 15.2 & -16.4 & -34.2 & -25.4 & -8.8 & -15.1 & -1.3 & TUCHOR & $?$ \\
\hline $25486 \ldots$ & & 37.3 & 18.8 & -20.2 & -13.8 & -10.9 & -10.2 & -15.1 & -8.2 & BETAPIC & BETAPIC? \\
\hline $25647 \ldots \ldots \ldots$ & AB Dor & 66.9 & 28.0 & 1.2 & -12.5 & -8.1 & -7.8 & -25.6 & -13.3 & ABDOR & ? \\
\hline $26373 \ldots \ldots \ldots \ldots \ldots \ldots \ldots \ldots \ldots \ldots$ & & 41.2 & 32.4 & -5.5 & -19.8 & -12.8 & -7.6 & -28.0 & -14.7 & ABDOR & ABDOR \\
\hline
\end{tabular}


TABLE 1-Continued

\begin{tabular}{|c|c|c|c|c|c|c|c|c|c|c|c|}
\hline HIP & Name & $\begin{array}{c}\Pi \\
(\mathrm{mas})\end{array}$ & $\begin{array}{c}\mathrm{RV} \\
\left(\mathrm{km} \mathrm{s}^{-1}\right)\end{array}$ & $\begin{array}{c}X \\
(\mathrm{pc})\end{array}$ & $\begin{array}{c}Y \\
(\mathrm{pc})\end{array}$ & $\begin{array}{c}Z \\
(\mathrm{pc})\end{array}$ & $\begin{array}{c}U \\
\left(\mathrm{~km} \mathrm{~s}^{-1}\right)\end{array}$ & $\begin{array}{c}V \\
\left(\mathrm{~km} \mathrm{~s}^{-1}\right)\end{array}$ & $\begin{array}{c}W \\
\left(\mathrm{~km} \mathrm{~s}^{-1}\right)\end{array}$ & $\begin{array}{c}\text { Assoc. } \\
\text { (Previous) }\end{array}$ & $\begin{array}{c}\text { Assoc. } \\
\text { (This Paper) }\end{array}$ \\
\hline $27321 \ldots \ldots \ldots \ldots \ldots \ldots \ldots \ldots$. & $\beta$ Pic & 51.9 & 20.0 & -3.3 & -16.3 & -9.8 & -11.0 & -15.9 & -9.1 & BETAPIC & BETAPIC? \\
\hline 28036 & & 18.5 & 24.3 & -21.1 & -43.6 & -24.4 & -11.7 & -21.3 & -5.8 & TUCHOR & BETAPIC? \\
\hline 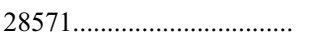 & HD 41067 & 3.9 & 33.9 & -148.2 & -188.0 & -95.6 & -11.4 & -30.9 & -13.0 & $?$ & ? \\
\hline 28921 & HD 41842 & 31.4 & 12.5 & -17.3 & -24.0 & -11.7 & -11.3 & -5.9 & -5.1 & $?$ & ? \\
\hline 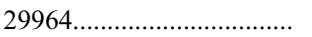 & HD 45081 & 26.0 & 15.0 & 7.4 & -33.1 & -18.2 & -10.7 & -15.4 & -8.2 & BETAPIC? & BETAPIC? \\
\hline 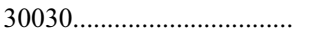 & HD 43989 & 20.1 & 19.0 & -41.6 & -26.3 & -7.6 & -10.0 & -18.6 & -5.3 & $?$ & ? \\
\hline 30034 & & 22.0 & 22.2 & -2.2 & -40.5 & -20.6 & -10.5 & -21.5 & -5.7 & TUCHOR & TUCHOR? BETAPIC? \\
\hline 30314 & & 42.6 & 31.2 & -0.2 & -21.0 & -10.6 & -7.7 & -27.7 & -14.2 & ABDOR & ABDOR? \\
\hline 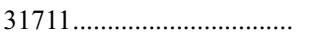 & & 46.2 & 32.3 & 0.4 & -19.6 & -9.2 & -7.2 & -28.9 & -14.8 & ABDOR & ABDOR \\
\hline 31878 & & 45.6 & 30.5 & 0.4 & -19.9 & -9.3 & -7.2 & -27.3 & -13.9 & ABDOR & ABDOR \\
\hline $32235 \ldots \ldots \ldots \ldots \ldots \ldots \ldots$ & & 17.7 & 19.9 & 11.2 & -49.4 & -25.0 & -11.7 & -22.8 & -5.1 & TUCHOR & UCL? \\
\hline 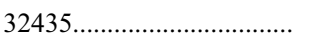 & & 17.5 & 12.5 & 22.5 & -45.8 & -26.1 & -8.6 & -19.5 & -0.7 & TUCHOR & TUCHOR \\
\hline 42794 & RS Cha & 10.2 & 26.0 & 34.8 & -83.9 & -36.0 & -8.1 & -27.6 & -14.1 & ETACHA & CHA \\
\hline $44458 \ldots \ldots \ldots \ldots \ldots \ldots \ldots \ldots \ldots$ & HD 77407 & 33.2 & 4.4 & -22.5 & -2.0 & 20.0 & -10.1 & -23.9 & -7.1 & $?$ & $?$ \\
\hline $48943 \ldots \ldots \ldots \ldots \ldots \ldots \ldots \ldots \ldots \ldots \ldots . .$. & & 5.2 & 39.0 & -31.2 & -172.7 & 78.6 & -24.5 & -36.4 & 5.7 & LCC? & LCC(runaway) \\
\hline 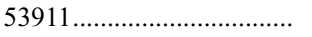 & TWA 1 & 17.7 & 12.7 & 7.8 & -51.4 & 22.0 & -12.8 & -18.8 & -7.0 & TWA & TWA \\
\hline 55505 & TWA 4 & 21.4 & 9.2 & 5.7 & -38.4 & 26.0 & -13.2 & -17.9 & -7.0 & TWA & TWA \\
\hline $55746 \ldots \ldots \ldots \ldots \ldots \ldots .$. & & 12.1 & 21.1 & 39.4 & -65.7 & -31.6 & -6.8 & -25.4 & -11.1 & CHA & CHA \\
\hline 57589 & TWA 9 & 19.9 & 9.5 & 15.2 & -43.5 & 20.2 & -7.1 & -14.9 & -3.1 & TWA & OPH? \\
\hline $57524 \ldots \ldots \ldots \ldots \ldots \ldots \ldots \ldots \ldots . .$. & TWA 19 & 9.6 & 11.5 & 38.9 & -94.1 & 21.1 & -8.9 & -17.7 & -6.0 & TWA & LCC \\
\hline 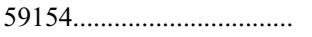 & RXJ 1207.9-7555 & 23.2 & -3.4 & 21.1 & -36.2 & -9.9 & -29.3 & -11.6 & -5.3 & CHA & $?$ \\
\hline $59960 \ldots \ldots \ldots \ldots \ldots \ldots \ldots \ldots \ldots . .$. & & 10.9 & 11.1 & 43.1 & -80.4 & 10.5 & -9.5 & -18.7 & -6.6 & LCC? & LCC \\
\hline 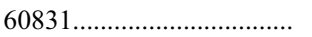 & HD 108574 & 25.5 & -1.5 & -8.9 & 8.5 & 37.2 & -27.9 & -18.2 & -4.1 & IC 2391 & ? \\
\hline $61498 \ldots \ldots \ldots \ldots \ldots \ldots \ldots \ldots \ldots \ldots .$. & TWA 11 & 14.9 & 9.4 & 30.6 & -53.7 & 26.1 & -8.5 & -18.3 & -3.6 & TWA & TWA? OPH? \\
\hline 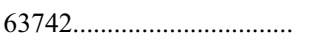 & HD 113449 & 45.2 & -5.8 & 7.4 & -9.3 & 18.7 & -7.7 & -25.4 & -16.4 & ABDOR & ABDOR? \\
\hline 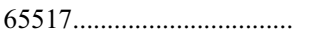 & & 9.6 & 10.0 & 63.4 & -78.7 & 25.6 & -9.8 & -22.1 & -3.0 & LCC? & UCL? \\
\hline $70350 \ldots \ldots \ldots \ldots \ldots \ldots \ldots \ldots \ldots$ & & 8.7 & 7.3 & 85.1 & -71.5 & 31.5 & -5.3 & -20.1 & -4.6 & UCL? & US? TUCHOR? \\
\hline $71631 \ldots \ldots \ldots \ldots \ldots \ldots \ldots$. & EK Dra & 29.5 & -20.6 & -5.9 & 21.4 & 25.6 & -7.2 & -29.0 & -4.7 & $?$ & ? \\
\hline 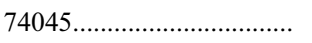 & & 34.0 & -1.0 & -9.1 & 21.3 & 18.2 & -27.4 & -8.5 & -5.4 & IC 2391 & IC 2391 \\
\hline 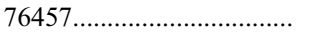 & & 6.7 & -26.5 & 131.3 & -60.4 & 36.2 & -33.7 & -16.4 & -14.2 & UCL? & $?$ \\
\hline 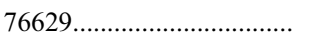 & HD 139084 & 25.1 & 0.5 & 32.1 & -23.5 & -1.3 & -10.4 & -14.6 & -9.7 & BETAPIC? & BETAPIC? TWA? \\
\hline 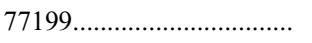 & KWLup & 24.4 & -4.9 & 36.8 & -12.0 & 13.3 & -9.6 & -21.0 & -7.4 & $?$ & TUCHOR(L)? \\
\hline $82569 \ldots \ldots \ldots \ldots \ldots \ldots \ldots \ldots . .$. & & 6.7 & -0.1 & 144.6 & -36.9 & 8.7 & -4.0 & -16.0 & -3.2 & UCL? & US? TUCHOR? \\
\hline $82688 \ldots \ldots \ldots$ & & 21.0 & -16.5 & 42.3 & 10.9 & 19.1 & -5.5 & -28.6 & -12.7 & ABDOR & ? \\
\hline $84586 \ldots \ldots \ldots \ldots \ldots \ldots \ldots \ldots \ldots . .$. & HD 155555A & 31.8 & 4.2 & 24.7 & -17.3 & -8.8 & -9.4 & -16.6 & -8.8 & BETAPIC & BETAPIC \\
\hline $86346 \ldots \ldots \ldots \ldots \ldots \ldots \ldots \ldots \ldots .$. & & 40.8 & -26.7 & -0.1 & 20.8 & 13.1 & -5.1 & -23.9 & -12.2 & ABDOR & ? \\
\hline 87819 & HD 163296 & 8.2 & 0.3 & 120.9 & 15.4 & 3.2 & 3.4 & -22.9 & -7.5 & $?$ & ? \\
\hline 88399 & HD 164249 & 21.3 & 0.1 & 43.2 & -14.3 & -11.3 & -7.4 & -15.6 & -8.9 & BETAPIC? & BETAPIC \\
\hline $92024 \ldots \ldots \ldots \ldots \ldots \ldots \ldots$ & & 34.2 & 2.0 & 23.3 & -13.1 & -11.8 & -10.9 & -15.5 & -9.3 & BETAPIC & BETAPIC \\
\hline $92680 \ldots \ldots \ldots \ldots \ldots \ldots \ldots \ldots . .$. & PZ Tel & 20.1 & -0.1 & 45.1 & -11.1 & -17.6 & -7.6 & -16.4 & -8.9 & TUCHOR? & BETAPIC \\
\hline 93815 .............................. & $\rho \mathrm{Tel}$ & 19.1 & 2.0 & 46.4 & -12.8 & -20.8 & -10.8 & -23.9 & -14.3 & TUCHOR? & $?$ \\
\hline 95264 & HR 7329 & 21.0 & 13.0 & 40.9 & -12.6 & -21.0 & 2.2 & -18.9 & -13.9 & BETAPIC & $?$ \\
\hline $95270 \ldots \ldots \ldots$ & & 19.8 & 0.2 & 43.3 & -13.4 & -22.4 & -9.2 & -16.5 & -8.5 & BETAPIC & BETAPIC \\
\hline 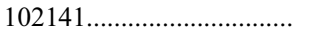 & AT Mic & 97.8 & -3.5 & 8.1 & 1.6 & -6.1 & -9.3 & -15.9 & -10.6 & BETAPIC & BETAPIC \\
\hline
\end{tabular}


TABLE 1-Continued

\begin{tabular}{|c|c|c|c|c|c|c|c|c|c|c|c|}
\hline HIP & Name & $\begin{array}{c}\Pi \\
\text { (mas) }\end{array}$ & $\begin{array}{c}\mathrm{RV} \\
\left(\mathrm{km} \mathrm{s}^{-1}\right)\end{array}$ & $\begin{array}{c}X \\
(\mathrm{pc})\end{array}$ & $\begin{array}{c}Y \\
(\mathrm{pc})\end{array}$ & $\begin{array}{c}Z \\
(\mathrm{pc})\end{array}$ & $\begin{array}{c}U \\
\left(\mathrm{~km} \mathrm{~s}^{-1}\right)\end{array}$ & $\begin{array}{c}V \\
\left(\mathrm{~km} \mathrm{~s}^{-1}\right)\end{array}$ & $\begin{array}{c}W \\
\left(\mathrm{~km} \mathrm{~s}^{-1}\right)\end{array}$ & $\begin{array}{c}\text { Assoc. } \\
\text { (Previous) }\end{array}$ & $\begin{array}{c}\text { Assoc. } \\
\text { (This Paper) }\end{array}$ \\
\hline 102409 & AU Mic & 100.6 & -4.9 & 7.8 & 1.7 & -6.0 & -10.4 & -16.5 & -10.2 & BETAPIC & BETAPIC \\
\hline 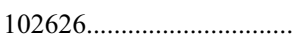 & Speedy Mic & 22.5 & -6.5 & 34.7 & 3.8 & -27.5 & -7.1 & -17.0 & -0.8 & $?$ & TUCHOR? \\
\hline 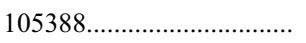 & & 21.8 & -1.0 & 32.0 & -9.0 & -31.6 & -7.9 & -20.5 & -0.7 & TUCHOR? & TUCHOR \\
\hline 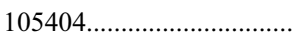 & BS Indi & 21.7 & 10.8 & 32.1 & -8.6 & -31.8 & -0.4 & -23.9 & -9.6 & $?$ & $?$ \\
\hline $107947 \ldots \ldots \ldots$ & & 22.2 & 1.4 & 28.1 & -15.9 & -31.5 & -9.0 & -19.5 & -0.2 & TUCHOR & TUCHOR \\
\hline $108195 \ldots \ldots \ldots \ldots \ldots \ldots \ldots \ldots \ldots .$. & & 21.5 & 1.0 & 28.8 & -16.3 & -32.7 & -9.6 & -19.8 & 0.1 & TUCHOR & TUCHOR \\
\hline 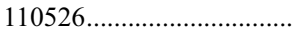 & & 62.2 & -20.6 & -0.0 & 15.0 & -5.7 & -6.9 & -27.8 & -15.2 & ABDOR & ABDOR \\
\hline 113579 & & 31.2 & 6.1 & 11.8 & 6.5 & -29.1 & -3.1 & -26.7 & -13.9 & ABDOR & ABDOR \\
\hline $114066 \ldots \ldots \ldots \ldots \ldots \ldots \ldots \ldots \ldots \ldots .$. & & 40.1 & -23.5 & -9.2 & 23.1 & 1.5 & -6.8 & -27.0 & -16.0 & ABDOR & ABDOR \\
\hline $114530 \ldots \ldots \ldots \ldots \ldots \ldots . .$. & & 19.8 & 9.1 & 21.9 & -6.2 & -45.1 & -8.6 & -28.9 & -10.4 & ABDOR & $?$ \\
\hline 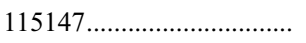 & V368 Cep & 50.7 & -17.0 & -9.0 & 16.6 & 5.7 & -10.0 & -23.7 & -5.5 & $?$ & $?$ \\
\hline 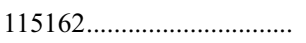 & & 20.3 & -19.7 & -12.4 & 45.4 & -14.8 & -4.7 & -27.3 & -14.2 & ABDOR & ABDOR? \\
\hline 116748 & & 21.6 & 7.7 & 21.3 & -23.5 & -33.6 & -9.3 & -25.8 & 1.6 & TUCHOR? & ? \\
\hline $118008 \ldots \ldots \ldots \ldots \ldots \ldots \ldots \ldots \ldots \ldots .$. & & 45.3 & 12.1 & 6.0 & -1.8 & -21.2 & -7.7 & -27.9 & -12.4 & ABDOR & ABDOR \\
\hline
\end{tabular}


TABLE 2

Phase Space Parameters for Nearby Clusters, Associations, and Star-forming Clouds

\begin{tabular}{|c|c|c|c|c|c|c|c|c|c|c|c|c|}
\hline Name & $\begin{array}{c}l \\
(\mathrm{deg})\end{array}$ & $\begin{array}{c}b \\
(\operatorname{deg})\end{array}$ & $\begin{array}{c}\mu_{l *} \\
\left(\operatorname{mas~yr}^{-1}\right)\end{array}$ & 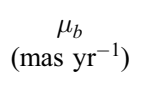 & $\begin{array}{c}d \\
(\mathrm{pc})\end{array}$ & $\begin{array}{c}\mathrm{RV} \\
\left(\mathrm{km} \mathrm{s}^{-1}\right)\end{array}$ & $\begin{array}{c}X \\
(\mathrm{pc})\end{array}$ & $\begin{array}{c}Y \\
(\mathrm{pc})\end{array}$ & $\begin{array}{c}Z \\
(\mathrm{pc})\end{array}$ & $\begin{array}{c}U \\
\left(\mathrm{~km} \mathrm{~s}^{-1}\right)\end{array}$ & $\begin{array}{c}V \\
\left(\mathrm{~km} \mathrm{~s}^{-1}\right)\end{array}$ & $\begin{array}{c}W \\
\left(\mathrm{~km} \mathrm{~s}^{-1}\right)\end{array}$ \\
\hline Blanco 1 ................................... & 14.22 & -79.02 & -1.0 & -19.4 & 269 & 3.6 & 49.7 & 12.6 & -264.1 & -22.6 & -7.0 & -8.2 \\
\hline IC 4665 & 30.61 & 17.08 & -7.2 & -3.0 & 352 & -10.3 & 289.6 & 171.3 & 103.4 & -1.1 & -14.6 & -7.8 \\
\hline IC 4756 & 36.38 & 5.25 & -5.4 & -2.2 & 330 & -25.8 & 264.6 & 194.9 & 30.2 & -15.4 & -21.9 & -5.8 \\
\hline Stephenson $1 \ldots \ldots \ldots \ldots \ldots \ldots$ & 66.85 & 15.51 & -3.6 & -0.8 & 315 & -21.6 & 119.3 & 279.1 & 84.2 & -3.1 & -21.0 & -6.9 \\
\hline 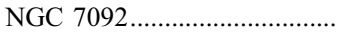 & 92.46 & -2.28 & -19.1 & -9.1 & 349 & -5.4 & -15.0 & 348.4 & -13.9 & 31.8 & -4.6 & -14.8 \\
\hline Lac OB 1 & 100.00 & -15.00 & -2.3 & -3.4 & 368 & -13.3 & -61.7 & 350.1 & -95.2 & 6.4 & -13.5 & -2.3 \\
\hline Сep OB $2 \ldots \ldots \ldots \ldots$ & 102.00 & 5.00 & -4.1 & -0.5 & 615 & -21.4 & -127.4 & 599.3 & 53.6 & 16.1 & -18.2 & -3.3 \\
\hline Cep OB 6 & 105.00 & 0.00 & 15.9 & -4.4 & 270 & -20.0 & -69.9 & 260.8 & 0.0 & -14.5 & -24.6 & -5.6 \\
\hline Alpha Persei............................. & 147.37 & -6.31 & 33.5 & -8.7 & 185 & -0.2 & -154.9 & 99.1 & -20.3 & -15.0 & -25.3 & -7.6 \\
\hline 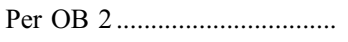 & 157.50 & -20.00 & 8.4 & -2.3 & 318 & 23.5 & -276.1 & 114.4 & -108.8 & -24.2 & -3.7 & -11.3 \\
\hline MBM 12 & 159.20 & -34.10 & 10.3 & -3.3 & 65 & -0.5 & -50.3 & 19.1 & -36.4 & -0.2 & -3.3 & -0.6 \\
\hline 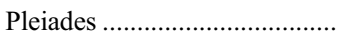 & 166.62 & -23.57 & 44.9 & -20.9 & 132 & 5.7 & -117.7 & 28.0 & -52.8 & -6.5 & -27.3 & -14.3 \\
\hline 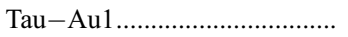 & 170.89 & -21.49 & 14.4 & -6.3 & 150 & 16.4 & -137.8 & 22.1 & -55.0 & -15.1 & -8.0 & -10.2 \\
\hline Tau-Au2 .................................. & 173.01 & -23.05 & 15.6 & -4.3 & 156 & 15.5 & -142.5 & 17.5 & -61.1 & -14.3 & -9.9 & -9.0 \\
\hline Tau-Au3 .................................... & 173.39 & -12.58 & 15.4 & -14.7 & 115 & 14.1 & -111.5 & 12.9 & -25.0 & -12.9 & -7.0 & -10.9 \\
\hline Orion Cloud A-B ..................... & 205.90 & -17.70 & -0.2 & -1.9 & 450 & 21.8 & -385.6 & -187.3 & -136.8 & -17.8 & -8.1 & -10.5 \\
\hline Praesepe …….......................... & 206.07 & 32.33 & -0.6 & -38.5 & 180 & 34.5 & -137.0 & -67.0 & 96.5 & -42.2 & -20.1 & -9.4 \\
\hline 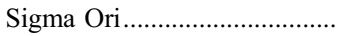 & 206.80 & -17.30 & 0.8 & -0.3 & 440 & 29.5 & -375.0 & -189.4 & -130.8 & -24.2 & -14.1 & -9.4 \\
\hline 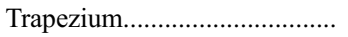 & 208.90 & -19.20 & 0.9 & 1.4 & 438 & 27.6 & -362.1 & -199.9 & -144.0 & -22.8 & -14.7 & -6.3 \\
\hline NGC 2232............................ & 214.33 & -7.73 & 0.7 & -5.2 & 365 & 21.0 & -298.7 & -204.0 & -49.1 & -15.5 & -12.1 & -11.7 \\
\hline Coma Ber & 221.28 & 84.03 & 6.6 & -12.9 & 81 & -0.1 & -6.3 & -5.6 & 80.6 & -2.0 & -5.1 & -0.6 \\
\hline 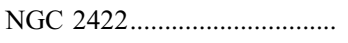 & 230.98 & 3.13 & -5.1 & -5.3 & 498 & 29.4 & -313.1 & -386.3 & 27.2 & -28.3 & -15.8 & -10.9 \\
\hline Collinder 121 ........................... & 236.00 & -8.00 & -5.1 & -1.5 & 543 & 26.0 & -300.7 & -445.8 & -75.6 & -25.0 & -13.6 & -7.4 \\
\hline 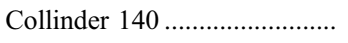 & 245.20 & -7.85 & -7.4 & -5.5 & 375 & 22.4 & -155.8 & -337.2 & -51.2 & -20.7 & -13.4 & -12.7 \\
\hline Collinder $135 \ldots \ldots \ldots \ldots \ldots \ldots \ldots$ & 248.76 & -11.20 & -10.3 & -6.8 & 300 & 16.4 & -106.6 & -274.3 & -58.3 & -18.8 & -7.9 & -12.7 \\
\hline NGC 2451............................... & 252.40 & -6.75 & -24.1 & -11.9 & 302 & 28.9 & -90.7 & -285.9 & -35.5 & -41.0 & -15.0 & -20.3 \\
\hline Vel OB 2 & 262.00 & -8.00 & -10.4 & -1.3 & 411 & 18.0 & -56.6 & -403.0 & -57.2 & -22.5 & -14.5 & -5.0 \\
\hline Trumpler 10 .................................. & 262.82 & 0.63 & -14.3 & -4.9 & 424 & 25.0 & -53.0 & -420.6 & 4.7 & -31.7 & -21.3 & -9.6 \\
\hline 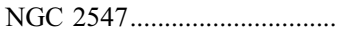 & 264.60 & -8.55 & -8.7 & -5.5 & 455 & 14.4 & -42.3 & -447.9 & -67.6 & -19.9 & -10.7 & -13.9 \\
\hline 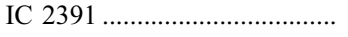 & 270.36 & -6.89 & -33.1 & -6.0 & 150 & 14.1 & 0.9 & -148.9 & -18.0 & -23.4 & -13.6 & -5.9 \\
\hline NGC 2516............................... & 273.86 & -15.89 & -11.6 & 1.5 & 346 & 22.7 & 22.4 & -332.0 & -94.7 & -17.5 & -23.7 & -3.8 \\
\hline vdB-Hagen 99 ........................... & 286.56 & -0.63 & -13.1 & -6.4 & 500 & 12.0 & 142.5 & -479.2 & -5.5 & -26.4 & -20.2 & -15.3 \\
\hline IC 2602 & 289.63 & -4.89 & -20.5 & 1.5 & 162 & 16.2 & 54.2 & -152.0 & -13.8 & -9.4 & -20.6 & -0.2 \\
\hline
\end{tabular}


TABLE 2-Continued

\begin{tabular}{|c|c|c|c|c|c|c|c|c|c|c|c|c|}
\hline Name & $\begin{array}{c}l \\
(\mathrm{deg})\end{array}$ & $\begin{array}{c}b \\
(\operatorname{deg})\end{array}$ & 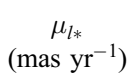 & $\begin{array}{c}\mu_{b} \\
\left(\operatorname{mas} \mathrm{yr}^{-1}\right)\end{array}$ & $\begin{array}{c}d \\
(\mathrm{pc})\end{array}$ & $\begin{array}{c}\mathrm{RV} \\
\left(\mathrm{km} \mathrm{s}^{-1}\right)\end{array}$ & $\begin{array}{c}X \\
(\mathrm{pc})\end{array}$ & $\begin{array}{c}Y \\
(\mathrm{pc})\end{array}$ & $\begin{array}{c}Z \\
(\mathrm{pc})\end{array}$ & $\begin{array}{c}U \\
\left(\mathrm{~km} \mathrm{~s}^{-1}\right)\end{array}$ & $\begin{array}{c}V \\
\left(\mathrm{~km} \mathrm{~s}^{-1}\right)\end{array}$ & $\begin{array}{c}W \\
\left(\mathrm{~km} \mathrm{~s}^{-1}\right)\end{array}$ \\
\hline NGC $3532 \ldots \ldots \ldots \ldots \ldots . . . .$. & 289.64 & 1.43 & -12.0 & 0.6 & 405 & 3.1 & 136.1 & -381.3 & 10.1 & -20.7 & -10.6 & 1.2 \\
\hline$\eta$ Cha & 292.48 & -21.65 & -39.5 & -10.7 & 97 & 16.1 & 34.6 & -83.6 & -35.9 & -11.8 & -19.1 & -10.5 \\
\hline 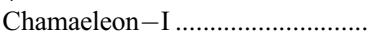 & 297.60 & -16.00 & -22.1 & -0.2 & 165 & 14.1 & 73.5 & -140.6 & -45.5 & -9.1 & -20.0 & -4.0 \\
\hline LCC & 298.00 & 7.00 & -32.1 & -13.1 & 118 & 12.0 & 55.0 & -103.4 & 14.4 & -9.8 & -19.7 & -5.8 \\
\hline 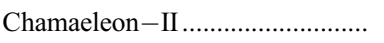 & 303.70 & -14.80 & -22.5 & -4.9 & 178 & 14.1 & 95.5 & -143.2 & -45.5 & -8.8 & -21.0 & -7.6 \\
\hline 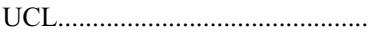 & 327.00 & 13.00 & -30.1 & -9.1 & 140 & 4.9 & 114.4 & -74.3 & 31.5 & -5.7 & -20.1 & -4.8 \\
\hline 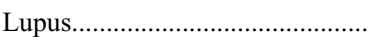 & 337.45 & 15.53 & -29.8 & -10.2 & 140 & 2.6 & 124.6 & -51.7 & 37.5 & -3.6 & -19.9 & -5.8 \\
\hline 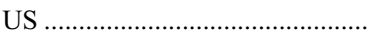 & 352.00 & 20.00 & -24.5 & -8.1 & 145 & -4.6 & 134.9 & -19.0 & 49.6 & -4.7 & -16.3 & -6.8 \\
\hline$\rho$ Oph & 352.95 & 16.69 & -22.8 & -9.0 & 135 & 2.2 & 128.3 & -15.9 & 38.8 & 1.9 & -14.9 & -4.9 \\
\hline LDN 1709 (Oph) ............................... & 354.43 & 16.35 & -26.3 & -7.2 & 160 & 2.2 & 152.8 & -14.9 & 45.0 & 1.7 & -20.2 & -4.6 \\
\hline NGC 6475 & 355.84 & -4.49 & -3.0 & -4.7 & 280 & -14.2 & 278.4 & -20.2 & -21.9 & -14.9 & -2.9 & -5.1 \\
\hline 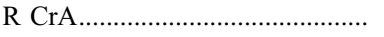 & 359.90 & -17.90 & -23.1 & -15.2 & 130 & -1.1 & 123.7 & -0.2 & -40.0 & -4.0 & -14.2 & -8.6 \\
\hline
\end{tabular}

Notes.-Abbreviated names: (US) Upper Scorpius; (UCL) Upper Centaurus Lupus; (LCC) Lower Centaurus Crux; (Tau-Au) Taurus-Auriga. The Tau-Au T-association, because of its large extents on the sky, is represented with three probable members, Tau-Au1-RX J0405.7+22; Tau-Au2-HIP 19176; and Tau-Au3-HIP 21852. 
TABLE 3

Encounters of HIP 14551 and HIP 102626 (=Speedy Mic) with Stars of TUCHOR Swarm

\begin{tabular}{|c|c|c|c|c|c|c|c|c|}
\hline \multirow[b]{2}{*}{ HIP } & \multicolumn{4}{|c|}{ HIP 14551} & \multicolumn{4}{|c|}{ HIP 102626} \\
\hline & $\begin{array}{l}D \text { Now } \\
(\mathrm{pc})\end{array}$ & $\begin{array}{l}D_{\min } \\
(\mathrm{pc})\end{array}$ & $\begin{array}{c}T_{\min } \\
(\mathrm{Myr})\end{array}$ & $\begin{array}{c}V_{\text {rel }} \\
\left(\mathrm{km} \mathrm{s}^{-1}\right)\end{array}$ & $\begin{array}{c}D \text { Now } \\
(\mathrm{pc})\end{array}$ & $\begin{array}{l}D_{\min } \\
(\mathrm{pc})\end{array}$ & $\begin{array}{c}T_{\min } \\
(\mathrm{Myr})\end{array}$ & $\begin{array}{c}V_{\text {rel }} \\
\left(\mathrm{km} \mathrm{s}^{-1}\right)\end{array}$ \\
\hline 1113 & 45.9 & 15.2 & -19.5 & 2 & & & & \\
\hline 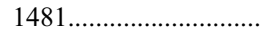 & & & & & 30.3 & 4.2 & -9.0 & 3 \\
\hline $2485 \ldots \ldots \ldots \ldots \ldots \ldots \ldots$ & & & & & 36.2 & 9.6 & -3.5 & 10 \\
\hline $2578 \ldots \ldots \ldots \ldots$ & & & & & 33.2 & 8.4 & -6.5 & 5 \\
\hline $6485 \ldots \ldots \ldots$ & 32.1 & 7.1 & -11.0 & 3 & 35.2 & 5.9 & -14.5 & 2 \\
\hline 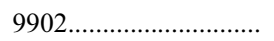 & & & & & 40.7 & 9.4 & -8.5 & 4 \\
\hline 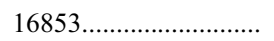 & 25.1 & 4.7 & -12.5 & 2 & 49.3 & 1.4 & -13.5 & 4 \\
\hline 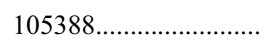 & & & & & 13.7 & 4.7 & -3.5 & 4 \\
\hline
\end{tabular}

Encounter of HIP 14551 and Speedy Mic

\begin{tabular}{|c|c|}
\hline $102626 \ldots \ldots \ldots \ldots \ldots \ldots$ & 64.7 \\
\hline
\end{tabular}

TABLE 4

EnCOUnters of TUCHOR Members with HIP 70350 and HIP 82569 OF US

\begin{tabular}{|c|c|c|c|c|c|c|c|c|}
\hline \multirow[b]{2}{*}{ HIP } & \multicolumn{4}{|c|}{ HIP 70350} & \multicolumn{4}{|c|}{ HIP 82569} \\
\hline & $\begin{array}{c}D \text { Now } \\
(\mathrm{pc})\end{array}$ & $\begin{array}{l}D_{\min } \\
(\mathrm{pc})\end{array}$ & $\begin{array}{c}T_{\min } \\
(\mathrm{Myr})\end{array}$ & $\begin{array}{c}V_{\text {rel }} \\
\left(\mathrm{km} \mathrm{s}^{-1}\right)\end{array}$ & $\begin{array}{c}D \text { Now } \\
(\mathrm{pc})\end{array}$ & $\begin{array}{l}D_{\min } \\
(\mathrm{pc})\end{array}$ & $\begin{array}{c}T_{\min } \\
(\mathrm{Myr})\end{array}$ & $\begin{array}{c}V_{\text {rel }} \\
\left(\mathrm{km} \mathrm{s}^{-1}\right)\end{array}$ \\
\hline 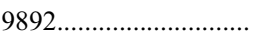 & 120.0 & 14.0 & -26.0 & 8 & & & & \\
\hline $9902 \ldots \ldots \ldots \ldots \ldots \ldots$ & 113.6 & 12.1 & -24.5 & 8 & 145.2 & 15.9 & -26.5 & 7 \\
\hline $32435 \ldots \ldots \ldots \ldots \ldots \ldots$ & & & & & 127.2 & 19.4 & -29.0 & 5 \\
\hline 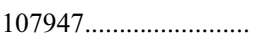 & 101.5 & 16.7 & -26.5 & 7 & 125.0 & 16.7 & -29.5 & 6 \\
\hline 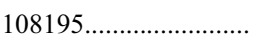 & 101.7 & 13.3 & -26.5 & 8 & & & & \\
\hline
\end{tabular}

TABLE 5

Encounters of HIP 30030 and HIP 92680 (=PZ Tel) with Stars of BETAPIC Swarm

\begin{tabular}{|c|c|c|c|c|c|c|c|c|}
\hline \multirow[b]{2}{*}{ HIP } & \multicolumn{4}{|c|}{ HIP 30030} & \multicolumn{4}{|c|}{ HIP 92680} \\
\hline & $\begin{array}{l}D \text { Now } \\
(\mathrm{pc})\end{array}$ & $\begin{array}{l}D_{\min } \\
(\mathrm{pc})\end{array}$ & $\begin{array}{c}T_{\min } \\
(\mathrm{Myr})\end{array}$ & $\begin{array}{c}V_{\text {rel }} \\
\left(\mathrm{km} \mathrm{s}^{-1}\right)\end{array}$ & $\begin{array}{l}D \text { Now } \\
(\mathrm{pc})\end{array}$ & $\begin{array}{l}D_{\min } \\
(\mathrm{pc})\end{array}$ & $\begin{array}{c}T_{\min } \\
(\mathrm{Myr})\end{array}$ & $\begin{array}{c}V_{\text {rel }} \\
\left(\mathrm{km} \mathrm{s}^{-1}\right)\end{array}$ \\
\hline 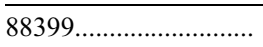 & 85.6 & 0.4 & -38.5 & 4 & & & & \\
\hline $10680 \ldots \ldots \ldots \ldots \ldots \ldots \ldots \ldots$ & & & & & 79.1 & 16.0 & -19.0 & 3 \\
\hline $11437 \ldots \ldots \ldots \ldots \ldots \ldots \ldots \ldots \ldots$ & & & & & 82.7 & 6.3 & -13.5 & 6 \\
\hline 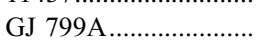 & 56.9 & 20.5 & -37.0 & 6 & & & & \\
\hline 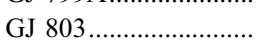 & & & & & 41.1 & 16.9 & -18.5 & 2 \\
\hline 84586 & & & & & 32.1 & 10.8 & -10.5 & 2 \\
\hline
\end{tabular}

TABLE 6

Encounters of BETAPIC Members with HIP 42794 and HIP 55746 of Chamaeleontis Group

\begin{tabular}{|c|c|c|c|c|c|c|c|c|}
\hline \multirow[b]{2}{*}{ HIP } & \multicolumn{4}{|c|}{ HIP 42794} & \multicolumn{4}{|c|}{ HIP 55746} \\
\hline & $\begin{array}{c}D \text { Now } \\
(\mathrm{pc})\end{array}$ & $\begin{array}{l}D_{\min } \\
(\mathrm{pc})\end{array}$ & $\begin{array}{c}T_{\min } \\
(\mathrm{Myr})\end{array}$ & $\begin{array}{c}V_{\text {rel }} \\
\left(\mathrm{km} \mathrm{s}^{-1}\right)\end{array}$ & $\begin{array}{c}D \text { Now } \\
(\mathrm{pc})\end{array}$ & $\begin{array}{l}D_{\min } \\
(\mathrm{pc})\end{array}$ & $\begin{array}{c}T_{\min } \\
(\mathrm{Myr})\end{array}$ & $\begin{array}{c}V_{\text {rel }} \\
\left(\mathrm{km} \mathrm{s}^{-1}\right)\end{array}$ \\
\hline $88399 .$. & 74.3 & 9.5 & -5.5 & 13 & 55.4 & 7.0 & -5.5 & 10 \\
\hline $84586 \ldots \ldots \ldots \ldots \ldots \ldots \ldots$ & & & & & 55.4 & 11.8 & -5.5 & 10 \\
\hline $92024 \ldots \ldots \ldots \ldots$ & 75.7 & 8.4 & -5.5 & 13 & 58.4 & 9.5 & -5.5 & 11 \\
\hline 92680 (=PZ Tel) ......... & & & & & 56.6 & 2.1 & -6.0 & 9 \\
\hline $95270 \ldots \ldots \ldots \ldots \ldots$ & & & & & 53.2 & 11.7 & -5.5 & 10 \\
\hline 76629............................. & 69.8 & 11.5 & -5.0 & 14 & & & & \\
\hline
\end{tabular}


TABLE 7

EnCounters of Young Stars with HIP 57589 (=TWA 9)

\begin{tabular}{crrrr}
\hline \hline HIP & $\begin{array}{c}D \text { Now } \\
(\mathrm{pc})\end{array}$ & $\begin{array}{c}D_{\min } \\
(\mathrm{pc})\end{array}$ & $\begin{array}{c}T_{\min } \\
(\mathrm{Myr})\end{array}$ & $\begin{array}{c}V_{\text {rel }} \\
\left(\mathrm{km} \mathrm{s}^{-1}\right)\end{array}$ \\
\hline 20390 (=TT Tau) $\ldots \ldots \ldots \ldots \ldots . .$. & 205.6 & 11.2 & -12.0 & 18 \\
$24947 \ldots \ldots \ldots \ldots . .$. & 56.3 & 10.4 & -23.5 & 4 \\
\hline
\end{tabular}

five core TUCHOR stars flew close by the stars HIP 70350 and 82569 (Tables 9 and 4), which are possible US members or stars ejected from that cloud at $4-5 \mathrm{~km} \mathrm{~s}^{-1}$. This leaves little doubt that the core of TUCHOR formed $27 \mathrm{Myr}$ ago in close proximity to US. The relative velocity with respect to US $\left(9.5 \mathrm{~km} \mathrm{~s}^{-1}\right)$ implies that the TUCHOR cloud was kinematically distinct from the greater US cloud. However, not all proposed TUCHOR members show traceback convergence, and some that do, have too recent encounters for the estimated age, for example, HIP 2485 and 6485 (Fig. 1). Undetected orbital motion perturbation cannot be ruled out, but another explanation is that TUCHOR comprises two subgroups with criss-crossing trajectories, but otherwise, of quite similar properties. The core TUCHOR member HIP 105388 was close to the four other candidates, HIP 490, 2485, 2578, and 6485 only $10,3.5,10$, and $9.5 \mathrm{Myr}$ ago, respectively, that is, at least $17 \mathrm{Myr}$ after the alleged formation time for these stars. Interestingly, none of the latter four have been close enough to the US progenitor cloud, but three of them, i.e., HIP 490, 2578, and 6485 , were proximate to the Lupus cloud $25 \mathrm{Myr}$ ago, flying by at $\approx 9 \mathrm{~km} \mathrm{~s}^{-1}$ (Table 12). The cloud in Lupus is a site of recent star formation, but it is not known when the first generation of stars was formed. James et al. (2006) determine an average age of $9.1 \pm 2.1 \mathrm{Myr}$ from model-dependent isochrone fitting for Lupus, but one star appears to match the $30 \mathrm{Myr}$ isochrone quite well in their Figure 3. A good deal of dispersed WTTS stars in Lupus are found close to a $25 \mathrm{Myr}$ isochrone in Wichmann et al. (1997 their Fig. 2). These apparently older stars have higher masses than their CTTS counterparts. The Lupus cloud and the stellar association are close spatially and kinematically to the Upper Centaurus Lupus (UCL) association, the latter being a relatively older formation where star formation has long ceased. The Lupus cloud is possibly a prominent outlying part on the fringes of the greater UCL complex that survived a major star formation event 25-27 Myr ago. The past trajectories of TUCHOR stars, US, and Lupus are depicted in Figure 5.

Two probably young stars, previously not assigned to any association, HIP 14551 and HIP 102626 (=BO Mic, Speedy Mic) were close together $13.5 \mathrm{Myr}$ ago, and surprisingly close to several TUCHOR members at various times during the past $14.5 \mathrm{Myr}$ (Table 3). Speedy Mic is a popular extremely active and rapidly rotating K dwarf. Anders et al. (1993) estimate its age at $14 \pm$ 7 Myr. The relative velocities are fairly small, suggesting a multiple system break-up or ejection from a cloud remnant. One pos-

TABLE 8

EnCounters of Young Stars with HIP 77199 (=KW LuP)

\begin{tabular}{|c|c|c|c|c|}
\hline HIP & $\begin{array}{c}D \text { Now } \\
(\mathrm{pc})\end{array}$ & $\begin{array}{l}D_{\min } \\
(\mathrm{pc})\end{array}$ & $\begin{array}{c}T_{\min } \\
(\mathrm{Myr})\end{array}$ & $\begin{array}{c}V_{\text {rel }} \\
\left(\mathrm{km} \mathrm{s}^{-1}\right)\end{array}$ \\
\hline $6485 \ldots \ldots$ & 62.5 & 8.8 & -30.5 & 9 \\
\hline $74045 \ldots \ldots$ & 56.9 & 3.9 & -2.5 & 22 \\
\hline 24244 (=८ Lep) & 106.1 & 18.3 & -21.0 & 5 \\
\hline $55746 \ldots \ldots \ldots$ & 70.1 & 13.8 & -10.0 & 7 \\
\hline $23200(=$ GJ 182) & 70.1 & 13.8 & -10.0 & 7 \\
\hline
\end{tabular}

TABLE 9

Encounters of Young Stars with the Upper Scorpius Association

\begin{tabular}{|c|c|c|c|c|c|}
\hline HIP & $\begin{array}{l}D \text { Now } \\
(\mathrm{pc})\end{array}$ & $\begin{array}{l}D_{\min } \\
(\mathrm{pc})\end{array}$ & $\begin{array}{c}T_{\min } \\
(\mathrm{Myr})\end{array}$ & $\begin{array}{c}V_{\text {rel }} \\
\left(\mathrm{km} \mathrm{s}^{-1}\right)\end{array}$ & Association \\
\hline $1113 \ldots \ldots \ldots \ldots \ldots . .$. & 140.3 & 29.1 & -27 & 8.7 & TUCHOR \\
\hline 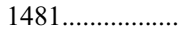 & 145.2 & 30.7 & -28 & 9.5 & TUCHOR \\
\hline 9141................... & 172.4 & 31.6 & -27 & 10.4 & TUCHOR \\
\hline $9892 .$. & 160.7 & 14.8 & -27 & 10.9 & TUCHOR \\
\hline $9902 \ldots \ldots \ldots \ldots \ldots . .$. & 154.3 & 3.6 & -26 & 10.1 & TUCHOR \\
\hline $32435 \ldots \ldots \ldots \ldots \ldots$ & 138.2 & 14.3 & -28 & 9.1 & TUCHOR \\
\hline $23200 \ldots \ldots \ldots \ldots \ldots$ & 169.7 & 20.5 & -8 & 20.6 & ? \\
\hline $24244 \ldots \ldots \ldots \ldots \ldots . .$. & 208.4 & 31.9 & -20 & 11.2 & TUCHOR(L) \\
\hline $28036 \ldots \ldots \ldots \ldots . .$. & 174.4 & 19.1 & -22 & 8.4 & BETAPIC? \\
\hline 30034 ................... & 155.5 & 17.1 & -22 & 8.0 & TUCHOR? \\
\hline 32235 ............... & 147.7 & 33.6 & -18 & 10.2 & ? \\
\hline 70350............... & 74.6 & 19.4 & -27 & 4.1 & US? \\
\hline $82569 \ldots \ldots \ldots \ldots \ldots$ & 45.7 & 15.0 & -28 & 5.0 & US? \\
\hline $107947 \ldots \ldots \ldots \ldots . . . .$. & 134.2 & 10.7 & -28 & 9.9 & TUCHOR \\
\hline $108195 \ldots \ldots \ldots \ldots . .$. & 134.4 & 9.4 & -27 & 10.5 & TUCHOR \\
\hline
\end{tabular}

sible scenario is that these two stars were components of a multiple system that remained in the core of TUCHOR until its disintegration about $14 \mathrm{Myr}$. In this case, the age of Speedy Mic is $\approx 27 \mathrm{Myr}$. A close conjunction of Speedy Mic with the Corona Australis ( $\mathrm{R} \mathrm{CrA}$ ) cloud is also found (Table 13), but this may be a mere chance, because such an early start of star formation in this currently active cloud is unlikely.

Two other isolated young stars, HIP $24244(=\iota$ Lep) and HIP 77199 (=KW Lup) have various signs of youth, but no unambiguous membership in any association. HIP 24244, a rapidly rotating B-type star, has a post-TT visual companion, but it is also an astrometric accelerating star (Makarov \& Kaplan 2005), making it at least a triple system. Due to its position on the sky, KW Lup could be suspected of being a member of the Lupus

TABLE 10

Encounters of Young Stars with the $\rho$ Oph Cloud

\begin{tabular}{|c|c|c|c|c|}
\hline HIP & $\begin{array}{c}D \text { Now } \\
(\mathrm{pc})\end{array}$ & $\begin{array}{l}D_{\min } \\
(\mathrm{pc})\end{array}$ & $\begin{array}{c}T_{\min } \\
(\mathrm{Myr})\end{array}$ & $\begin{array}{c}V_{\text {rel }} \\
\left(\mathrm{km} \mathrm{s}^{-1}\right)\end{array}$ \\
\hline $28036 \ldots$ & 164.6 & 29.1 & -11 & 14.6 \\
\hline $30034 \ldots$ & 145.5 & 30.7 & -11 & 13.7 \\
\hline $92680 \ldots \ldots \ldots \ldots \ldots \ldots \ldots$ & 100.7 & 7.7 & -9 & 11.0 \\
\hline $27321 \ldots \ldots$ & 140.4 & 28.6 & -10 & 13.2 \\
\hline 102141......................... & 129.5 & 27.9 & -10 & 12.1 \\
\hline $102409 \ldots \ldots \ldots \ldots \ldots \ldots$ & 129.8 & 18.0 & -9 & 13.1 \\
\hline 88399 & 98.8 & 15.2 & -9 & 10.4 \\
\hline $560 \ldots \ldots \ldots$ & 147.5 & 31.8 & -11 & 13.6 \\
\hline $76629 \ldots \ldots \ldots \ldots \ldots \ldots$ & 104.5 & 30.0 & -7 & 13.1 \\
\hline $10680 \ldots \ldots \ldots \ldots \ldots \ldots \ldots \ldots$ & 170.5 & 7.9 & -12 & 14.1 \\
\hline $11437 \ldots \ldots \ldots$ & 173.7 & 2.7 & -10 & 16.2 \\
\hline $21547 \ldots \ldots \ldots \ldots \ldots \ldots \ldots$ & 162.0 & 23.3 & -9 & 16.2 \\
\hline $84586 \ldots \ldots \ldots \ldots \ldots \ldots . .$. & 114.1 & 13.6 & -9 & 12.1 \\
\hline $92024 \ldots \ldots \ldots \ldots \ldots \ldots . .$. & 116.0 & 17.8 & -8 & 13.6 \\
\hline $95270 \ldots \ldots \ldots \ldots \ldots \ldots \ldots$ & 104.7 & 20.5 & -8 & 12.4 \\
\hline $53911 \ldots \ldots \ldots \ldots \ldots$ & 126.7 & 30.9 & -7 & 14.6 \\
\hline $55505 \ldots \ldots \ldots \ldots \ldots$ & 125.4 & 26.7 & -7 & 14.8 \\
\hline $23200 \ldots \ldots \ldots \ldots \ldots$ & 159.7 & 22.4 & -5 & 27.7 \\
\hline $30030 \ldots \ldots \ldots \ldots$ & 176.4 & 29.8 & -14 & 11.1 \\
\hline $24244 \ldots \ldots \ldots \ldots \ldots \ldots$ & 198.4 & 30.7 & -11 & 17.6 \\
\hline $19335 \ldots \ldots \ldots \ldots \ldots \ldots \ldots$ & 155.8 & 29.3 & -4 & 33.0 \\
\hline 77199 …………… & 95.1 & 30.9 & -7 & 12.9 \\
\hline $42794 \ldots \ldots \ldots \ldots \ldots \ldots \ldots$ & 137.7 & 10.0 & -7 & 19.3 \\
\hline $55746 \ldots \ldots \ldots \ldots \ldots \ldots \ldots$ & 123.9 & 19.1 & -8 & 15.6 \\
\hline
\end{tabular}


TABLE 11

Encounters of Young Stars with the LDN 1709 (Oph) Cloud

\begin{tabular}{|c|c|c|c|c|}
\hline HIP & $\begin{array}{l}D \text { Now } \\
(\mathrm{pc})\end{array}$ & $\begin{array}{l}D_{\min } \\
(\mathrm{pc})\end{array}$ & $\begin{array}{c}T_{\min } \\
(\mathrm{Myr})\end{array}$ & $\begin{array}{c}V_{\text {rel }} \\
\left(\mathrm{km} \mathrm{s}^{-1}\right)\end{array}$ \\
\hline 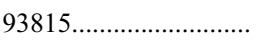 & 125.1 & 22.5 & -7 & 16.6 \\
\hline $23200 \ldots \ldots \ldots \ldots \ldots \ldots \ldots \ldots$ & 184.8 & 29.7 & -6 & 26.7 \\
\hline 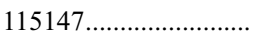 & 169.5 & 10.5 & -16 & 10.8 \\
\hline $77199 \ldots \ldots \ldots \ldots \ldots \ldots \ldots \ldots$ & 120.3 & 23.3 & -10 & 11.0 \\
\hline
\end{tabular}

star-forming region, but they are in fact separated by $\approx 100 \mathrm{pc}$ today and have significantly different velocities in $U$. And yet the origin of these stars may be related to the Lupus cloud (Table 12). Both stars were relatively close to Lupus (21-23 pc) several Myr later than the Lupus branch of TUCHOR, flying by at roughly the same velocity. KW Lup moved slower than the other stars with respect to the cloud, which is why it is still located in the same constellation. Whether these two stars belong to TUCHOR is questionable, but the passage near the Lupus cloud is a possible origin scenario. The star KW Lup may be younger than $18 \mathrm{Myr}$ (11 Myr according to Neuhäuser \& Brandner 1998). Significantly later encounters of KW Lup with the Ophiuchus clouds $\rho$ Oph and LDN 1709 (Tables 10 and 11) present an interesting alternative. In particular, the closer LDN 1709 passage 10 Myr ago matches well the previously quoted isochrone age. Significant fly-by velocities of $11-13 \mathrm{~km} \mathrm{~s}^{-1}$ show that KW Lup was not formed within the Ophiuchus clouds.

\section{THE BETA PICTORIS MOVING GROUP}

A group of young stars around the famous disk star $\beta$ Pic (hereafter BETAPIC), which is only $\approx 20$ pc away from the Sun, was suggested to be a comoving association by Barrado y Navascués et al. (1999) and Zuckerman et al. (2001b). An age of $\approx 20 \mathrm{Myr}$ was estimated from isochrones for the two late-type dwarfs AT Mic (HIP 102141) and AU Mic (HIP 102409) in a wide common proper motion pair. The list of proposed members gradually expanded to include up to 30 stellar systems. Later isochrone age estimates converged to $\approx 12 \mathrm{Myr}$. Table 1 lists 17 of the proposed members with phase space parameters deemed sufficiently reliable for this analysis. All pairwise encounters found between proposed members of BETAPIC are presented in Figure 2. The data for each individual event are not accurate enough for, e.g., a meaningful age estimation, but essential conclusions can be drawn from the whole data set as a statistical ensemble.

The association shows a modest degree of compression in the past, similar to TUCHOR discussed in the previous section. A fairly large "initial" size has been estimated by Ortega et al. (2002) by a similar traceback method, suggesting a gravitationally

TABLE 12

Encounters of Young Stars with the Lupus Cloud

\begin{tabular}{|c|c|c|c|c|}
\hline HIP & $\begin{array}{c}D \text { Now } \\
(\mathrm{pc})\end{array}$ & $\begin{array}{l}D_{\min } \\
(\mathrm{pc})\end{array}$ & $\begin{array}{c}T_{\min } \\
\text { (Myr) }\end{array}$ & $\begin{array}{c}V_{\text {rel }} \\
\left(\mathrm{km} \mathrm{s}^{-1}\right)\end{array}$ \\
\hline 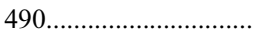 & 144.5 & 9.7 & -25 & 9.1 \\
\hline$\ldots \ldots \ldots \ldots \ldots$ & 135.1 & 20.3 & -25 & 9.0 \\
\hline 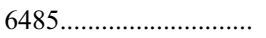 & 142.1 & 24.1 & -25 & 10.0 \\
\hline 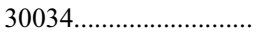 & 139.9 & 34.0 & -19 & 6.9 \\
\hline ….............. & 129.5 & 23.0 & -16 & 8.8 \\
\hline 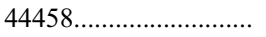 & 156.2 & 20.2 & -37 & 4.9 \\
\hline $24244 \ldots \ldots \ldots \ldots \ldots \ldots \ldots \ldots \ldots \ldots \ldots \ldots \ldots$ & 194.2 & 22.8 & -19 & 10.1 \\
\hline 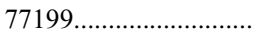 & 99.4 & 20.5 & -18 & 5.3 \\
\hline
\end{tabular}

TABLE 13

Encounters of Young Stars with the R CrA Cloud

\begin{tabular}{|c|c|c|c|c|}
\hline HIP & $\begin{array}{c}D \text { Now } \\
(\mathrm{pc})\end{array}$ & $\begin{array}{l}D_{\min } \\
(\mathrm{pc})\end{array}$ & $\begin{array}{c}T_{\min } \\
(\mathrm{Myr})\end{array}$ & $\begin{array}{c}V_{\text {rel }} \\
\left(\mathrm{km} \mathrm{s}^{-1}\right)\end{array}$ \\
\hline $92680 \ldots$. & 82.5 & 14.1 & -22 & 3.4 \\
\hline ................ & 120.8 & 25.6 & -20 & 5.8 \\
\hline $10680 \ldots$ & 154.1 & 31.6 & -20 & 6.1 \\
\hline $30034 \ldots$. & 139.9 & 34.0 & -19 & 6.9 \\
\hline $11437 \ldots$ & 157.6 & 26.5 & -16 & 8.6 \\
\hline . & 105.2 & 25.6 & -18 & 5.1 \\
\hline 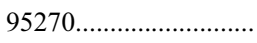 & 83.3 & 13.7 & -15 & 4.9 \\
\hline $102626 \ldots$ & 90.0 & 3.0 & -39 & 8.1 \\
\hline $19335 \ldots \ldots \ldots \ldots \ldots \ldots \ldots \ldots$ & 148.2 & 28.2 & -18 & 5.1 \\
\hline 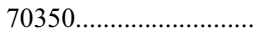 & 108.1 & 10.7 & -12 & 9.2 \\
\hline 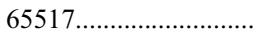 & 118.8 & 23.0 & -9 & 12.3 \\
\hline
\end{tabular}

unbound state at birth. The relative velocities at times of nearest approach (Fig. 2) are fairly small, around $2-3 \mathrm{~km} \mathrm{~s}^{-1}$, indicating a weak expansion. The scatter of encounter times is large, but it is evident that all times at the high end of the distribution, those longer than 60 Myr ago, involve only two stars, HIP 29964 and 27321 ( $\beta$ Pic). Excluding these two stars, the mean time of nearest approach is $-22 \pm 12 \mathrm{Myr}$. For all events shown in Figure 2, the mean time is $-31 \pm 21 \mathrm{Myr}$. The former result is in better agreement with the isochrone estimates.

Ortega et al. (2002) speculate that the relative proximity of BETAPIC to the US and the Lower Centaurus Crux (LCC) associations about $11 \mathrm{Myr}$ ago $(\approx 45 \mathrm{pc}$ on their estimation) could be the key to the mystery of its formation. A hypothetical supernova explosion at that time within one of these OB associations could trigger star formation in the passing BETAPIC cloud, in their opinion. It should be noted that this hypothesis implies that the star formation event in US or LCC started at least 15 Myr ago and that it was capable of generating $\mathrm{O}$ stars, which we do not find today. Such massive stars, before going off as supernovae, should have swept up the gas in these associations, precluding formation of new stars. According to the new data presented by Preibisch et al. (2002) the bulk of late-type members of US are as young as 5 Myr. Thus, there is a chronological difficulty in the supernova scenario. The passages near US and LCC were too distant by the criteria adopted in this paper to be recorded. But I find other encounter events for BETAPIC, which may be more plausible.

The list of close approaches of young stars to the $\rho$ Ophiuchus star-forming cloud in Table 10 is conspicuously dominated by the proposed BETAPIC members. A few stars previously assigned to TUCHOR that failed all previously discussed TUCHOR selection tests, appear now among the certified BETAPIC stars with conforming parameters. Therefore, the stars HIP 92680 (PZ Tel), HIP 28036, and HIP 30034 may be considerably younger than the assumed age of TUCHOR (28 Myr). The star HIP 30034 is significantly more metal poor $([\mathrm{Fe} / \mathrm{H}]=-0.64$ from Nordström et al. 2004) than bona fide TUCHOR stars $([\mathrm{Fe} / \mathrm{H}]=-0.25)$,

TABLE 14

Encounters of Young Stars with the Cepheus OB6 Association

\begin{tabular}{|c|c|c|c|c|}
\hline HIP & $\begin{array}{c}D \text { Now } \\
(\mathrm{pc})\end{array}$ & $\begin{array}{l}D_{\min } \\
(\mathrm{pc})\end{array}$ & $\begin{array}{c}T_{\min } \\
(\mathrm{Myr})\end{array}$ & $\begin{array}{c}V_{\text {rel }} \\
\left(\mathrm{km} \mathrm{s}^{-1}\right)\end{array}$ \\
\hline 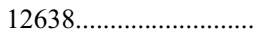 & 236.6 & 23.9 & -38 & 10.5 \\
\hline 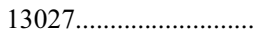 & 255.3 & 19.1 & -39 & 9.4 \\
\hline 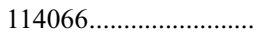 & 245.3 & 3.1 & -37 & 12.9 \\
\hline
\end{tabular}


TABLE 15

Other Possible Encounters of Young Stars with Clusters, Associations, and Clouds

\begin{tabular}{|c|c|c|c|c|c|}
\hline HIP & Counterpart & $\begin{array}{l}D \text { Now } \\
(\mathrm{pc})\end{array}$ & $\begin{array}{l}D_{\min } \\
(\mathrm{pc})\end{array}$ & $\begin{array}{c}T_{\min } \\
(\mathrm{Myr})\end{array}$ & $\begin{array}{c}V_{\text {rel }} \\
\left(\mathrm{km} \mathrm{s}^{-1}\right)\end{array}$ \\
\hline $20390 \ldots \ldots \ldots \ldots \ldots$ & TauAu1 & 30.2 & 16.3 & -2 & 12.6 \\
\hline 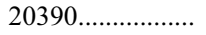 & TauAu2 & 23.3 & 7.0 & -1 & 12.0 \\
\hline 32235 ........................ & UCL & 120.3 & 19.7 & -20 & 6.9 \\
\hline $74045 \ldots \ldots \ldots \ldots \ldots$ & IC 2391 & 174.3 & 33.0 & -24 & 6.9 \\
\hline $74045 \ldots \ldots \ldots \ldots$ & UCL & 156.7 & 8.4 & -6 & 24.1 \\
\hline $74045 \ldots \ldots \ldots \ldots \ldots$ & Blanco 1 & 288.5 & 17.8 & -17 & 24.2 \\
\hline $74045 \ldots \ldots \ldots \ldots \ldots$ & NGC 2516 & 372.2 & 25.4 & -20 & 20.6 \\
\hline $53911 \ldots \ldots \ldots \ldots \ldots$ & LCC & 70.6 & 22.5 & -23 & 2.8 \\
\hline $55505 \ldots \ldots$ & LCC & 82.5 & 26.9 & -20 & 3.6 \\
\hline $57524 \ldots \ldots \ldots \ldots \ldots$ & Eta Cha & 58.1 & 28.6 & -8 & 7.3 \\
\hline $48943 \ldots \ldots \ldots \ldots \ldots$ & UCL & 181.9 & 30.9 & -6 & 26.5 \\
\hline $48943 \ldots \ldots \ldots \ldots \ldots$ & LCC & 127.9 & 5.6 & -5 & 25.3 \\
\hline $48943 \ldots \ldots \ldots \ldots \ldots$ & Eta Cha & 159.3 & 23.9 & -5 & 28.4 \\
\hline $28571 \ldots \ldots \ldots \ldots \ldots$ & UCL & 313.1 & 25.6 & -47 & 14.6 \\
\hline $28571 \ldots \ldots \ldots \ldots \ldots$ & Tau $\mathrm{Au} 1$ & 214.2 & 11.4 & -9 & 23.6 \\
\hline $28571 \ldots \ldots \ldots \ldots \ldots$ & Tau Au 2 & 208.4 & 23.3 & -9 & 21.8 \\
\hline 59154...................... & NGC 6475 & 258.1 & 6.5 & -19 & 13.1 \\
\hline $82569 \ldots \ldots \ldots \ldots \ldots$ & UCL & 53.1 & 28.5 & -12 & 4.4 \\
\hline $82569 \ldots \ldots \ldots \ldots \ldots$ & Alpha Per & 330.2 & 39.5 & -49 & 8.7 \\
\hline 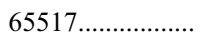 & UCL & 51.6 & 21.5 & -12 & 4.0 \\
\hline $59960 \ldots \ldots \ldots \ldots \ldots \ldots$ & LCC & 26.2 & 22.8 & -6 & 1.4 \\
\hline $59960 \ldots \ldots \ldots \ldots \ldots$ & Eta Cha & 47.3 & 12.3 & -8 & 6.0 \\
\hline
\end{tabular}

but close in metallicity to $\mathrm{PZ} \mathrm{Tel}([\mathrm{Fe} / \mathrm{H}]=-0.58)$. A few other young stars coming from Ophiuchus share this low metallicity, including HIP 76629, 84586, and 115147 , but the majority of BETAPIC members have nearly solar metallicities $([\mathrm{Fe} / \mathrm{H}] \approx$ -0.15). Mutual conjunction of PZ Tel and HIP 30034 with BETAPIC stars are presented in Table 5. Sufficiently small impact distances and moderate impact velocities justify the consignment of this stars to BETAPIC in Table 1. Thus, one of the criteria to discriminate TUCHOR and BETAPIC members in the "Assoc. This Paper" column is whether they passed near the US or the $\rho$ Oph clouds in the past.

Returning to Table 10, we notice that the mean time of encounters of BETAPIC stars with the $\rho$ Oph cloud is $-9.5 \mathrm{Myr}$, and the impact velocity is a substantial $13.3 \mathrm{~km} \mathrm{~s}^{-1}$. The time of encounter is in good agreement with the more recent estimates of the age of BETAPIC. However, just as in case of TUCHOR, I find a dual encounter for this stream. A few core members of BETAPIC, including AU Mic (=HIP 102409) and the newly acquired PZ Tel, had a fly-by near the star-forming cloud in Corona Australis around the deeply embedded star R CrA (Table 13). Ironically, the mean time of this event is $\approx-19 \mathrm{Myr}$, matching quite well the original estimation of age. Although a smaller number of stars are involved in the CrA encounter, it cannot be easily discarded as a random occurrence, because the impact velocity is significantly smaller in that case, and lower velocity impacts are more likely in the local domain of interstellar clouds, characterized by low velocity dispersions. Thus, the uncertainty

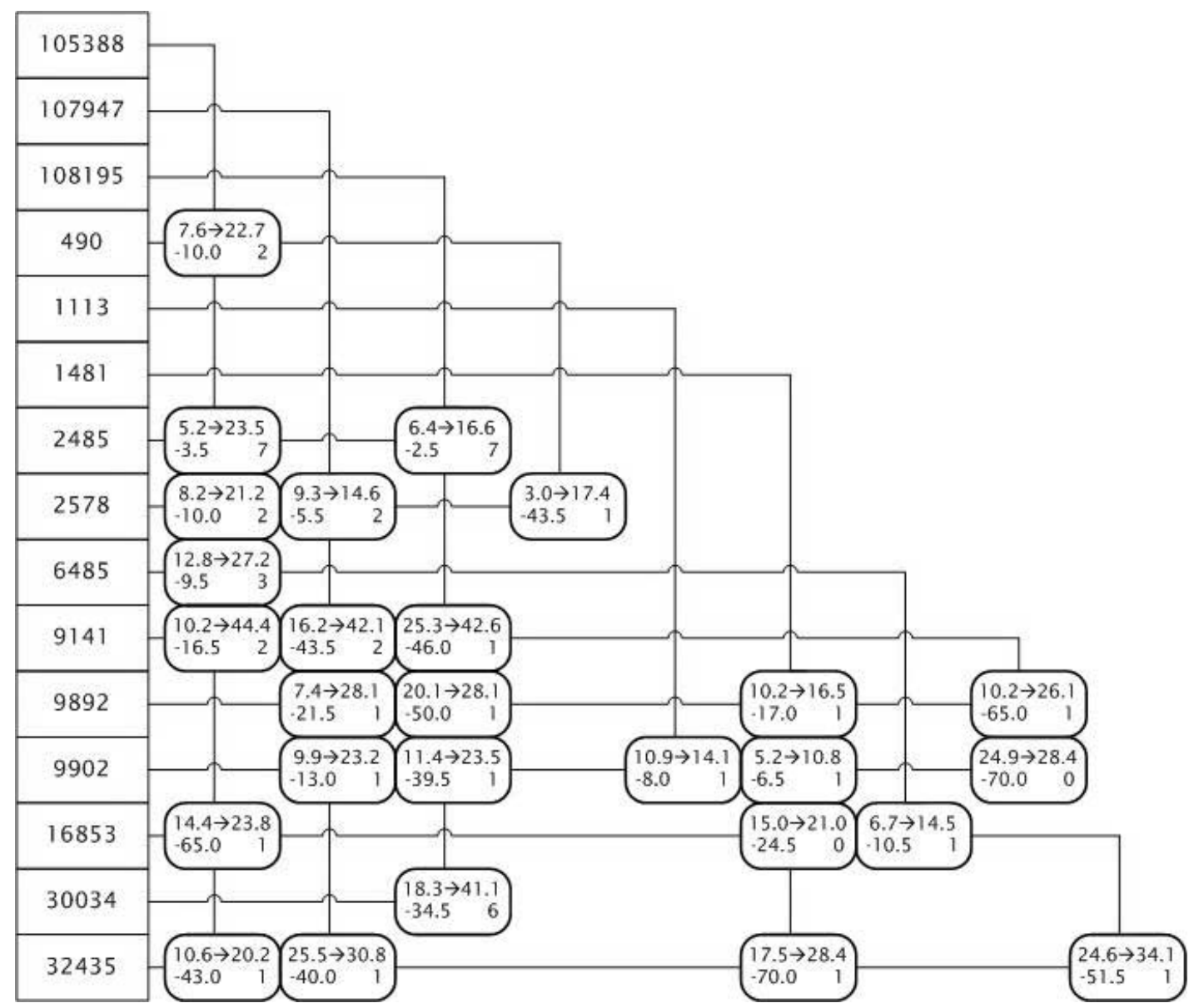

FIG. 1.-Matrix of pairwise encounters of probable members of TUCHOR. Each event is represented by a rectangle with rounded corners, connected by lines to the

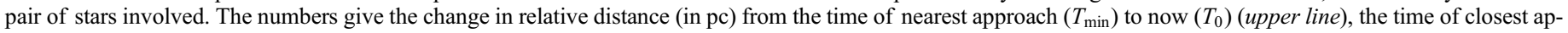
proach in Myr (lower left corner), and the relative velocity at $T_{\min }$ (lower right corner). Stars are listed in the left column of rectangular blocks by Hipparcos numbers. 


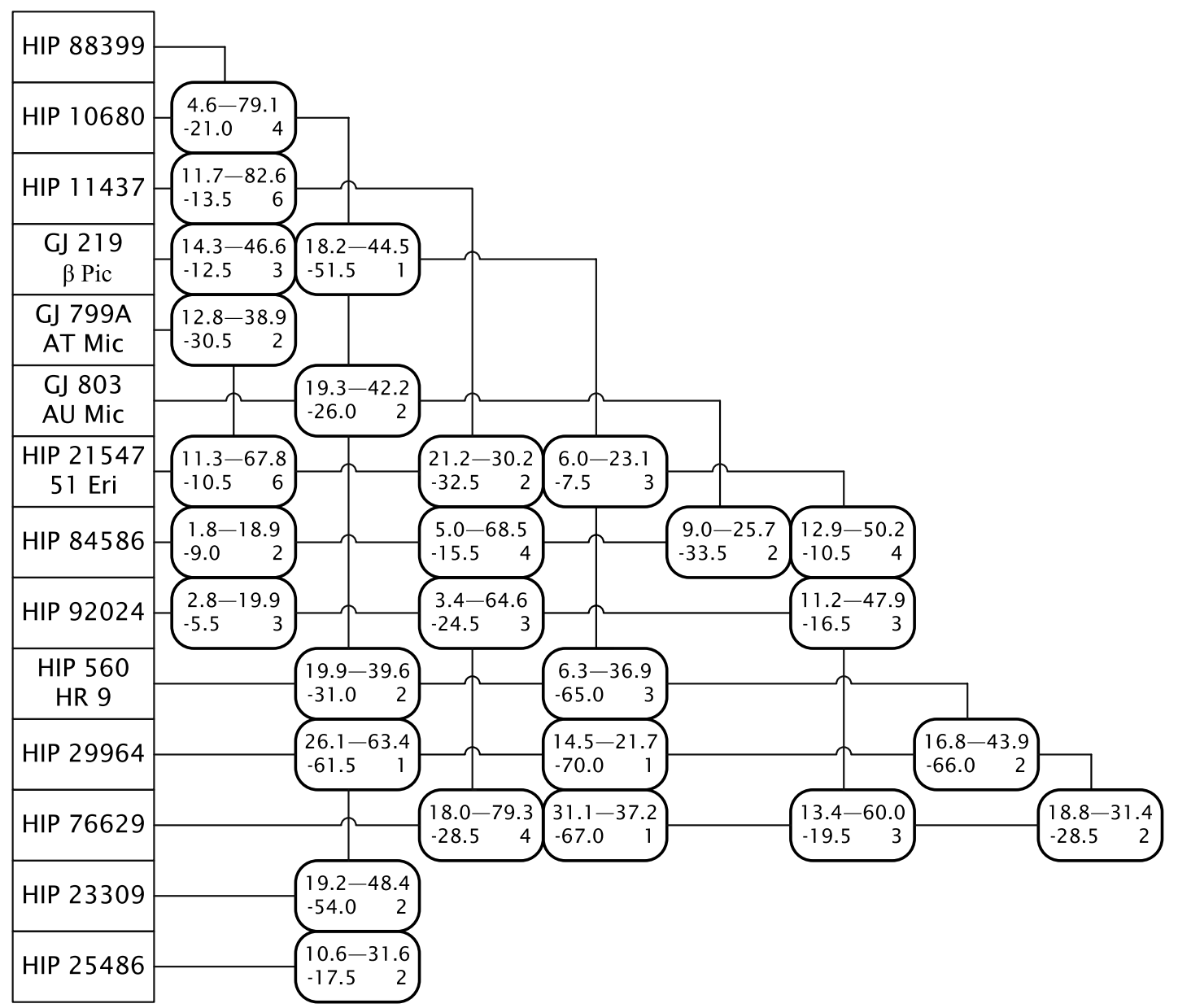

FIG. 2.-Matrix of pairwise encounters of probable members of BETAPIC. Notations are the same as in Fig. 1.

about the age and formation site of BETAPIC remains. It appears from the data in hand that the later high-velocity encounter with the $\rho$ Oph cloud is more likely to be responsible for the actual generation of stars, whereas the earlier conjunction may indicate that the progenitor BETAPIC cloud was a kinematically deviant part of the greater CrA complex (Fig. 6).

The two stars representing the so-called Chamaeleon-Near group, HIP 42794 (RS Cha) and HIP 55746, were also near the $\rho$ Oph cloud 7-8 Myr ago (Table 10), traveling at high velocities. Furthermore, these two very young stars underwent close, welldefined encounters with stars in the BETAPIC stream at $t=$

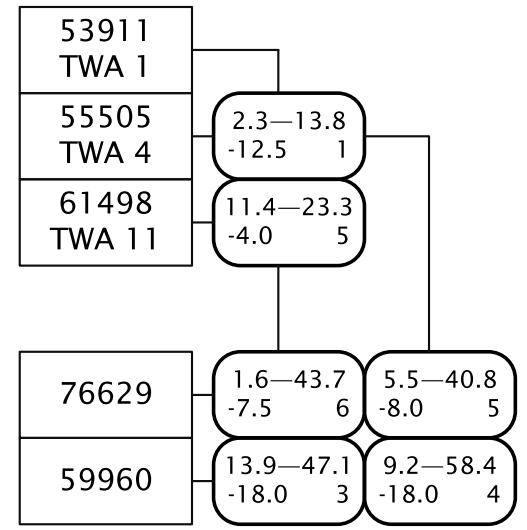

Fig. 3.- Matrix of pairwise encounters of probable members of TWA. Notations are the same as in Fig. 1.
-5.5 Myr (Table 6). On the other hand, no encounters of these stars with the more distant Chamaeleon I and Chamaeleon II starforming regions have been found. Therefore, this important (but poorly represented in my analysis) group may be related to the Ophiuchus rather than Chamaeleon clouds, owing its name to a chance sky projection. The inferred age is $8 \mathrm{Myr}$, but it certainly needs a more detailed investigation.

\section{THE TW HYA ASSOCIATION (TWA)}

The small group of T Tauri stars around TW Hya (TWA 1 or HIP 53911) is historically first and probably the youngest of the nearby swarms of pre-main-sequence stars. Unfortunately, trigonometric parallaxes are available for only several of the originally proposed members (Rucinski \& Krautter 1983; de la Reza et al. 1989; Gregorio-Hetem et al. 1992; Webb et al. 1999), which is why only five TWA stars are included in Table 1. Furthermore, two stars out of these five have been identified as nonmembers. HIP 57524 (TWA 19) is only 20 pc away from the center of the Lower Centaurus Crux OB association (LCC), and it moves slowly $\left(\approx 2 \mathrm{~km} \mathrm{~s}^{-1}\right)$ with respect to LCC. This star, therefore, belongs to LCC (see also Lawson \& Crause 2005). The star HIP 57589 (TWA 9) has a velocity vector in disagreement with the rest of the group and is likely an interloper (de la Reza et al. 2006). The list of mutual encounters for this group is short (Fig. 3) including only two events. The sample is too small to draw conclusions, but the times of the two events match the range of previous expansion age estimates from the literature, from 5 to $12 \mathrm{Myr}$, depending on the method and selection of members (Makarov \& Fabricius 2001; Makarov et al. 2005; de la Reza et al. 2006). 


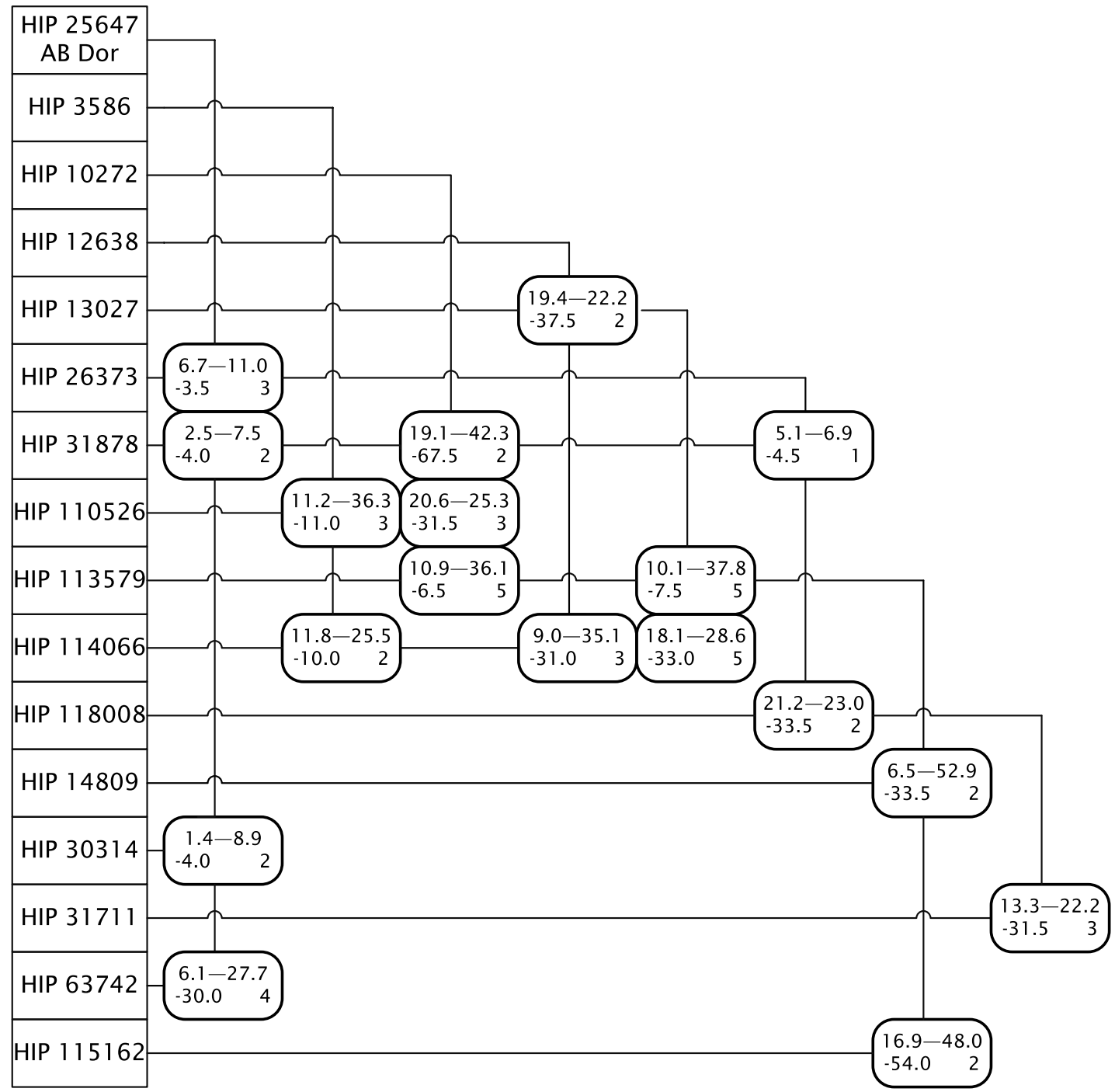

FIG. 4.- Matrix of pairwise encounters of probable members of ABDOR. Notations are the same as in Fig. 1.

Encounters of TWA with two other stars of note are also shown in Figure 3. The close conjunction of the two certified TWA members, TWA 1 and TWA 4, with the star HIP 76629 is surprising, because the latter star has been included in the BETAPIC swarm by substantial evidence. On the other hand, its kinematic parameters fit the TWA orbit so well that it has been identified as possible TWA member in Makarov et al. (2005). These encounters took place 7.5-8 Myr ago, in good agreement with the commonly adopted age of TWA, but significantly later than the birth of BETAPIC. The low metallicity $([\mathrm{Fe} / \mathrm{H}]=-0.75)$ also differentiates this star from the majority of BETAPIC members. Apart from this star of uncertain status, there are other common properties between the two groups. Both BETAPIC and TWA swarms passed near the Ophiuchus association in the past (Table 10), TWA first and BETAPIC somewhat later. These approaches were not positionally coincident either. It appears that the progenitor clouds of TWA and BETAPIC were separate parts of a large and turbulent complex that experienced a cascade high-velocity impact with the Ophiuchus complex between 11 and 7 Myr ago.

It has been suggested that the fly-by of TWA near the LCC OB association could be responsible for the small-scale star formation burst in the former. Indeed, encounters of TWA 1 and TWA 4 with the bona fide LCC member HIP 59960 (Fig. 3) and with the center of this association (Table 15) at moderate relative ve- locities are detected. In all these cases the times are rather too early for the age of TWA (between 18 and $23 \mathrm{Myr}$ ). This points at Ophiuchus as the more likely origin of TWA, but the kinematic alignment of TWA and LCC deserves further investigation. It may be considered that the progenitor TWA cloud was a stray part of the LCC cloud, which did not generate stars until the subsequent impact with the Ophiuchus complex.

My data support the earlier conclusion that HIP 57589 (TWA 9) is nonmember. It was never closer to any of TWA members than it is today. The origin of this young star becomes an open issue. The only two conjunctions with other young stars found here are listed in Table 7. The high velocity fly-by with T Tauri is apparently a statistical fluke, but the conjunction with HIP 2494723.5 Myr ago deserves attention because of the moderate relative velocity. The latter star was proposed as a member of TUCHOR, but it fails all the kinematic criteria of such affiliation, probably because of its higher $V$ velocity.

\section{THE ABDOR MOVING GROUP}

This group of comoving stars was proposed by Zuckerman et al. (2004). The prototype star AB Dor (HIP 25647) is a relatively well-studied nearby $(\approx 15 \mathrm{pc})$ dwarf, one of the famous three rotators with outstanding signs of activity (BO Mic, PZ Tel, and $\mathrm{AB}$ Dor). The age of the group is roughly estimated from 


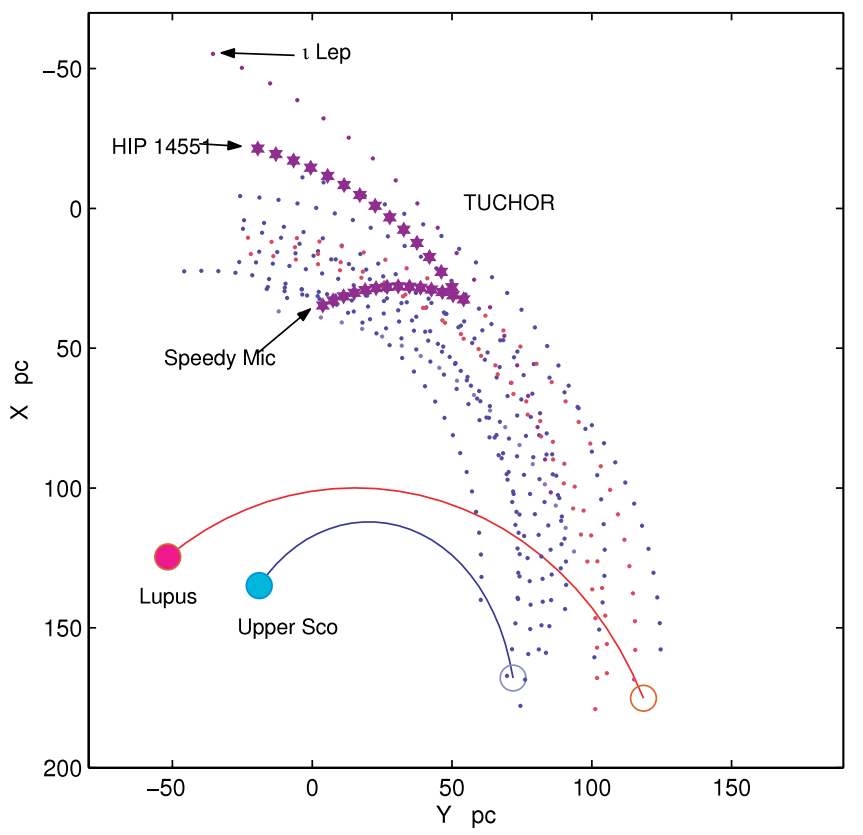

FIG. 5.- Trajectories of TUCHOR stars, Upper Sco OB association, and Lupus T-association for the past $27 \mathrm{Myr}$, and of the stars Speedy Mic and HIP 14551 for the past $13 \mathrm{Myr}$. Current positions of stars are in the upper left corner; the estimated locations of the Lupus and US associations today are indicated with filled circles, and their locations $27 \mathrm{Myr}$ ago with open circles. The tracks of the stars HIP 14551 and Speedy Mic (BO Mic) are shown with asterisks $\left(^{*}\right)$. A solar velocity of $\left(U_{\odot}, V_{\odot}, W_{\odot}\right)=(9.0,13.4,8.3) \mathrm{km} \mathrm{s}^{-1}$ is adopted from Torra et al. (2000).

isochrones at 50 Myr. Thus, it appears to be an older analog of the TUCHOR and TWA swarms in the southern sky. The membership and the age of this group was subsequently analyzed by (López-Santiago et al. 2006). They conclude that some of the originally proposed members belong to the B4 kinematic subgroup, which is considerably older. ABDOR may be the oldest association we are dealing with in this paper, pushing the limits of applicability of the kinematic method in use. Extrapolated over long intervals of time, the convergence of 3D tracks is perturbed by errors in the input radial velocities and parallaxes and by unknown orbital motion in binaries. Indeed, we find relatively few individual approach events for this populous group in Figure 4. All but one encounters of the star AB Dor with other alleged ABDOR members appear to be very recent events ( $<4 \mathrm{Myr})$, in disagreement with the estimated age of the group, raising doubts about the status of this star. It is noted that AB Dor is a longperiod astrometric binary star (Guirado et al. 1997; Makarov \& Kaplan 2005; Nielsen et al. 2005; Guirado et al. 2006) whose radial velocity may be affected by the orbital motion around the center of gravity. On the other hand, other studies suggest an older age of 75-150 Myr for the AB Dor multiple system (Luhman \& Potter 2006), raising doubts about its membership in the ABDOR group. Excluding the star AB Dor, the mean epoch of individual encounters is $-28 \pm 19 \mathrm{Myr}$. The median time is -32 Myr. Hence, first clues are obtained that the average age of the indicated stars may be younger than the original estimate.

The velocity vector of ABDOR is distinct from the younger swarms we have considered, having markedly smaller $V$ and $W$ components. It is not surprising that backtracking its orbit points at a quite different domain in the local part of the Galaxy as the likely origin of this association. Remarkably, no intersections of proposed ABDOR members are found with other young stars from Table 1, nor with the Sco-Cen associations or the neighboring clouds in Lupus, Ophiuchus, and Corona Australis. Instead, I

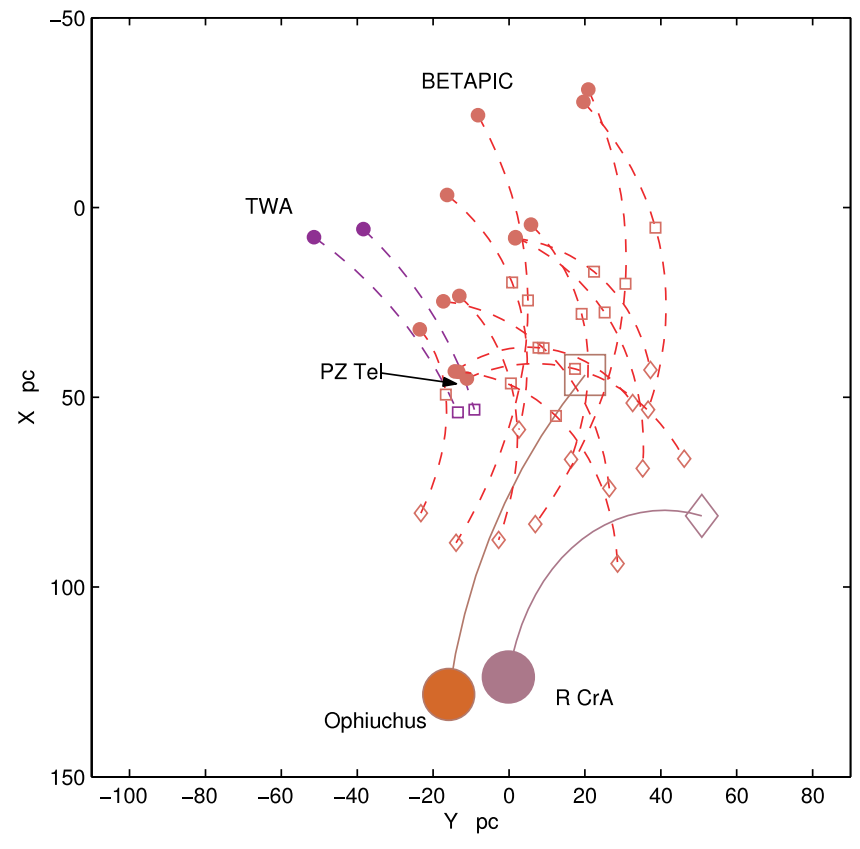

FIG. 6.- Trajectories of BETAPIC and TWA stars, and the R CrAT-association for the past $20 \mathrm{Myr}$, and of the Ophiuchus SFR for the past 9 Myr. Positions of stars and associations today are shown with filled circles, 9 Myr ago with open squares, and $27 \mathrm{Myr}$ ago with open diamonds. Note the extended shape of the BETAPIC group at all three snapshots.

find three encounters with the Cepheus OB6 association (Table 14) at $-38 \pm 1$ Myr. Pairwise encounters between these three stars (HIP 12638, 13027, and 114066) are also close to this time. With more accurate observational data, more encounters of the ABDOR nucleus with this OB association are likely to be found. Interestingly, the time of this encounter matches the age (rather uncertain) of Cepheus OB6 (de Zeeuw et al. 1999). It is therefore proposed that the ABDOR nucleus formed 38 Myr ago during a close passage of, or encounter with, the Cepheus OB6 cloud, which may have triggered formation of the latter association as well.

\section{OTHER ENCOUNTERS}

Other past conjunctions of young stars with nearby star-forming regions, clusters, and associations are listed in Table 15. This includes events that are not clearly associated with the kinematical history of the groups discussed in the previous sections. It begins with the T Tauri star, the prototype of the class, commonly referred to the Taurus-Auriga (TAUAU in this paper) star-forming region. Although two recent fly-bys with the fairly reliable TAUAU members RX J0405.7+22 ( Tau-Au1) and HIP 19176 ( Tau-Au2) are detected, these results cast some doubt on the origin of T Tauri because of the high relative velocities $\left(12.6\right.$ and $12.0 \mathrm{~km} \mathrm{~s}^{-1}$, respectively). Several conjectures could be considered, including that $\mathrm{T}$ Tauri is not related to the association of post-TT stars in TAUAU. Additional observational data on the TAUAU association and a dedicated study are needed to shed light on this intriguing problem.

It is not uncommon that isolated young stars of unknown origin are involved in multiple encounters and fly-bys with clusters and associations. It appears that there is a dispersed stream of stars threading together most of the diverse complexity of the local Gould Belt system (Torra et al. 2000). The star HIP 74045 is a typical representative of this stream. Previously associated with the IC 2391 supercluster in Montes et al. (2001), it experienced a variety of encounter events between 24 and 6 Myr ago. 
The closest encounter is possibly that with the Upper Centaurus Lupus (UCL) OB association only $6 \mathrm{Myr}$ ago, but the more distant conjunction with the young open cluster IC 2391 is the more plausible origin event because of the much lower departing velocity. Stars are ejected from clusters mainly through chance interactions with hard massive binaries or disintegration of nonhierarchical multiple systems (trapezia); both mechanisms cannot impart velocities higher than several $\mathrm{km} \mathrm{s}^{-1}$ on the ejected stars. This result supports the proposed association of HIP 74045 with the IC 2391 cluster.

The well-known exception from the dynamical preference of low relative velocities is the phenomenon of runaway stars. These stars are ejected from their native clusters or associations at high velocities, often in excess of $20 \mathrm{~km} \mathrm{~s}^{-1}$. Table 15 includes one well-defined runaway event discovered by Hoogerwerf et al. (2001), that of the star HIP 48943 ejected from the LCC association $5 \mathrm{Myr}$ ago at $25.3 \mathrm{~km} \mathrm{~s}^{-1}$. I find, in addition, that this ejection was close in space and in time to the emergence of the $\eta$ Chamaeleontis (Eta Cha) minicluster from the LCC association, discovered by Mamajek et al. (1999, 2000). Could these two dramatic events inside LCC be related? The commonly accepted explanation of runaway stars assumes a binary or multiple system of stars, whose most massive component goes off as a supernova. The suddenly disrupted gravitational bond shoots the less massive companion as a sling. As supernova explosions normally occur in binary systems, this scenario does not require any additional dynamical processes to take place. However, this paradigm meets considerable difficulties at HIP 48943, which is an astrometric binary itself, probably with an orbital period of several years. It is not clear how this binary could survive the recoil disruption.

\section{DISCUSSION}

It follows from the present analysis of Galactic tracks that the majority of nearby young stars were formed during close passages or encounters of their natal clouds with other cloud complexes that are situated today at somewhat larger distances. These triggered star formation events usually resulted in small comoving groups of $20-40$ stars, rarely including more massive members than A0. Although the groups were generated over a relatively short time span (a few Myr), their initial extent in space could reach $20-40 \mathrm{pc}$; we find no evidence for significant expansion except for the youngest TWA group. Furthermore, the initial configuration of some of these groups appear to have markedly prolate or linearly extended, rather than round-shaped forms (Figs. 5 and 6).

These results are consistent with the paradigm of short-lived, highly dynamical star-forming cores, which rapidly emerge from coalescing flows of gas and rapidly dissipate after a short burst of star generation (Hartmann et al. 2001). This scenario is opposed to quasi-static dynamically relaxed cores that remain intact for tens of Myr and produce stars, which can be dynamically ejected. Coalescing large-scale flows can almost simultaneously produce new stars over large dimensions in space in linear or sheetlike structures. Chance confluence of two flows or interaction of two expanding supernova shells are usually considered. It can be added that a grazing encounter of two cold molecular clouds at moderate velocities can quickly produce an extended, shortlived core and generate a small number of stars without sweeping up or dispersing the bulk of either cloud. It may be an important clue to understanding of the recent star formation that the $\mathrm{OB}$ and $T$ associations in the Scorpius-Centaurus, Ophiuchus, Lupus, Corona Australis, and Chamaeleon have all similar velocities in space. They may be dynamically separate parts of a large supercloud, which, on their way around the Galactic center, come into interaction with each other time and time again, producing bursts of newborn stars. The Local Association (e.g., Montes et al. 2001), a large conglomerate of young stars, associations, and clusters ranging in age between 5 and $200 \mathrm{Myr}$, may be the result of an internally interacting, kinematically aligned stream of clouds.

The research described in this paper was carried out at the Jet Propulsion Laboratory, California Institute of Technology, under a contract with the National Aeronautics and Space Administration. This research has made use of the SIMBAD database, operated at CDS, Strasbourg, France.
Anders, G. J., et al. 1993, MNRAS, 265, 941

Asiain, R., Figueras, F., \& Torra, J. 1999, A\&A, 350, 434

Barrado y Navascués, D., et al. 1999, ApJ, 520, L123

de la Reza, R., Jilinsky, E., \& Ortega, V. G. 2006, AJ, 131, 2609

de la Reza, R., et al. 1989, ApJ, 343, L61

de Zeeuw, P. T., Hoogerwerf, R., de Bruijne, J. H. J., Brown, A. G. A., \& Blaauw, A. 1999, AJ, 117, 354

Dolan, C. J., \& Mathieu, R. D. 2001, AJ, 121, 2124

Feigelson, E. D. 1996, ApJ, 468, 306

Feigelson, E. D., Lawson, W. A., \& Garmire, G. P. 2003, ApJ, 599, 1207

Fukushige, T., \& Heggie, D. C. 2000, MNRAS, 318, 753

Gregorio-Hetem, J., et al. 1992, AJ, 103, 549

Guirado, J. C., et al. 1997, ApJ, 490, 835 2006, A\&A, 446, 733

Hartmann, L., Ballesteros-Paredes, J., \& Bergin, E. 2001, ApJ, 562, 852 Høg E., et al. 2000, A\&A, 355, L27

Hoogerwerf, R., de Bruijne, J. H. J., de Zeeuw, P. T. 2001, A\&A, 365, 49

James, D. J., et al. 2006, A\&A, 446, 971

Jilinsky, E., Ortega, V. G., de la Reza, R. 2005, ApJ, 619, 945

Lawson, W. A., \& Crause, L. A. 2005, MNRAS, 357, 1399

López-Santiago, J., et al. 2006, ApJ, 643, 1160

Luhman, K. L., \& Potter, D. 2006, ApJ, 638, 887

Makarov, V. V., \& Fabricius, C. 2001, A\&A, 368, 866

Makarov, V. V., Gaume, R. A., \& Andrievsky, S. M. 2005, MNRAS, 362, 1109

Makarov, V. V., \& Kaplan, G. H. 2005, AJ, 129, 2420

Makarov, V. V., Olling, R. P., \& Teuben, P. J. 2004, MNRAS, 352, 1199

Makarov, V. V., \& Urban, S. E. 2001, in ASP Conf. Ser. 244, Young Stars Near

Earth: Progress and Prospects, ed. R. Jayawardhana \& Th. Greene (San

Francisco: ASP), 57
REFERENCES

Mamajek, E. E., Lawson, W. A., \& Feigelson, E. D. 1999, ApJ, 516, L77 . 2000, ApJ, 544, 356

Montes, D., et al. 2001, MNRAS, 328, 45

Neuhäuser, R., \& Brandner, W. 1998, A\&A, 330, L29

Nielsen, E. L., et al. 2005, Astron. Nachr., 326, 1033

Nordström, B., et al. 2004, A\&A, 418, 989

Ortega, V. G., et al. 2002, ApJ, 575, L75

Preibisch, T., et al. 2002, AJ, 124, 404

Robichon, N., Arenou, F., Mermilliod, J.-C., \& Turon, C. 1999, A\&A, 345, 471

Rucinski, S. M., \& Krautter, J. 1983, A\&A, 121, 217

Sterzik, M. F., \& Durisen, R. H. 1995, A\&A, 304, L9

Strassmeier, K. G., et al. 2000, A\&AS, 142, 275

Torra, J., Fernández, D., \& Figueras, F. 2000, A\&A, 359, 82

Torres, C. A. O., et al. 2000, AJ, 120, 1410

Torres, G., et al. 2003, AJ, 125, 825

Webb, R. A., et al. 1999, ApJ, 512, L63

Wichmann, R., Schmitt, J. H. M. M., \& Hubrig, S. 2003, A\&A, 399, 983

Wichmann, R., et al. 1997, A\&A, 320, 185

Weinberger, A. J., et al. 2000, ApJ, 544, 937

Zacharias, N., et al. 2004, AJ, 127, 3043

Zuckerman, B., \& Song, I. 2004, ARA\&A, 42, 685

Zuckerman, B., Song, I., \& Bessell, M. S. 2004, ApJ, 613, L65

Zuckerman, B., Song, I., \& Webb, R. A. 2001a, ApJ, 559, 388

Zuckerman, B., \& Webb, R. A. 2000, ApJ, 535, 959

Zuckerman, B., et al. 2001b, ApJ, 562, L87 